\title{
Wnt/ $\beta$-catenin activation epigenetically reprograms Regulatory T cells in IBD and progression to dysplasia.
}

Fotini Gounari ( $\nabla$ fgounari@uchicago.edu )

University of Chicago https://orcid.org/0000-0003-2936-066X

Jasmin Quandt

University of Chicago

Stephen Arnovitz

University of Chicago

Leila Haghi

University of Chicago

Janine Woehlk

University of Chicago

Azam Moshin

University of Chicago

Michael Okoreeh

University of Chicago

Priya Mathur

University of Chicago

Akinola Emmanuel

University of Chicago

Abu Osman

University of Chicago

Manisha Krishnan

University of Chicago

Samuel Morin

University of Chicago

Alexander Pearson

University of Chicago

Randy Sweis

University of Chicago https://orcid.org/0000-0002-0497-9637

Joel Pekow

University of Chicago

Christopher Weber 
University of Chicago

Khashayarsha Khazaie

Mayo Clinic College of Medicine and Graduate School

\section{Article}

Keywords: Regulatory T cells, IBD

Posted Date: December 1st, 2020

DOI: https://doi.org/10.21203/rs.3.rs-110212/v1

License: (c) (1) This work is licensed under a Creative Commons Attribution 4.0 International License. Read Full License 


\section{Abstract}

The molecular and functional diversity of regulatory T-cells $\left(T_{\text {reg }} s\right)$ in health and in disease remains unclear. We previously described in colorectal cancer (CRC) patients a subpopulation of RORyt ${ }^{+} \mathrm{T}_{\text {reg }} \mathrm{s}$ with elevated expression of $\beta$-catenin and pro-inflammatory properties. Here we observed progressive expansion of RORyt ${ }^{+} \mathrm{T}_{\text {reg }} \mathrm{s}$ in inflammatory bowel disease (IBD) patients during inflammation and early dysplasia. Activating Wnt/ $\beta$-catenin signaling in human and murine $T_{\text {reg }} s$ was sufficient to recapitulate the disease-associated increase in frequencies of RORyt ${ }^{+} T_{\text {reg }}$ s expressing IL-17, IFN- $\gamma$, and TNFa. We found that binding of the $\beta$-catenin interacting partner, TCF-1, to DNA overlapped with Foxp3 binding at enhancer sites of pro-inflammatory pathway genes. Sustained Wnt/ $\beta$-catenin activation induced newly accessible chromatin sites in these genes and upregulated their expression. These findings indicate that

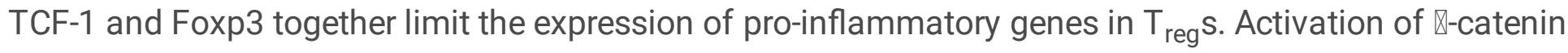
signaling interferes with this function and promotes the disease-associated RORyt ${ }^{+} \mathrm{T}_{\text {reg }}$ phenotype.

\section{Introduction}

Regulatory $T$ cells $\left(T_{\text {reg }} s\right)$ are essential to sustain peripheral tolerance and prevent autoimmune pathology. A growing body of evidence suggests that $\mathrm{T}_{\text {reg }} \mathrm{s}$ adapt phenotypically and functionally to their anatomic location and in response to the inflammatory millieu ${ }^{1,2}$. Multiple $T_{\text {reg }}$ sub-populations have been described that express additional $T$ helper $\left(T_{H}\right)$ cell lineage transcription factors ${ }^{3}$ including T-bet $^{4}$, GATA-3 ${ }^{5}$, and RORYt ${ }^{6,7}$. Adopting these programs equips $T_{\text {reg }} s$ with the ability to specifically regulate different $T_{H}$ responses. The intestine comprises a highly diverse $T_{\text {reg }}$ compartment. Specifically, RORytexpressing $T_{\text {reg }} s$ are found in the colon, small intestine (SI), and at lower frequencies in the peripheral lymphoid organs of healthy mice $6,7,8$. These cells are necessary to maintain the balance of intestinal inflammatory responses. In humans, however, it remains unclear whether RORyt ${ }^{+} /$Foxp $^{+} \mathrm{T}_{\text {reg }}$ s have physiological regulatory functions.

$T_{\text {reg }}$ s are defined by a core regulatory program established during late $T_{\text {reg }}$ differentiation by Foxp3 binding to gene enhancers made accessible by Foxo $1^{9}$. Moreover, Foxp3 acts together with other factors forming multi-molecular complexes with different modes of action ${ }^{10,11}$; functioning as an activator in complex with RelA, Ikzf2 (Helios), Ep300, and Kat5, and a repressor with EZH2, YY1, and Ikzf3 ${ }^{12}$. This seminal work ${ }^{9}, 10,11,12$, however, suggests that Foxp3 has a complex interactome, which requires further study. Recently, it was suggested that overlap of Foxp3 binding with the transcription factor TCF-1 may affect the maintenance of $T_{\text {reg }}$ function ${ }^{13}$. We further showed that TCF-1 binding also overlaps with multiple other factors including Ikaros, Runx, Ets, and HEB in double positive thymocytes. In particular, it cooperates with HEB to initiate T cell-specific programs through active enhancer (AE) binding and regulation of chromatin accessibility ${ }^{14}$. Furthermore, TCF-1 plays critical roles in the differentiation of $T_{H}$ lineages ${ }^{15}$, memory formation ${ }^{16}$, and counteracting exhaustion ${ }^{17}$. In T cells, TCF- 1 is the bona fide DNA- 
binding partner of $\beta$-catenin, the central effector of the Wnt-signaling cascade. The classical Wntsignaling dogma defines TCF-1 as a DNA-bound repressor until Wnt signals stabilize $\beta$-catenin, which translocates to the nucleus and binds TCF-1 to initiate transcription ${ }^{18}$. $\beta$-catenin is also stabilized upon T cell receptor (TCR) engagement in T cells ${ }^{19}$. Excessive $W n t / \beta$-catenin activation in $T_{\text {reg }} s$ may impact $T_{\text {reg }}$ function ${ }^{20}$ and is linked to autoimmunity in the context of multiple sclerosis ${ }^{21}$. Foxp3 binding to DNA has been suggested to overlap with $\beta$-catenin in human $T_{\text {reg }} \mathrm{s}$, which parallels the overlap between TCF- 1 and Foxp3 DNA occupancy in mice ${ }^{13}$.

Immune suppression through recruitment of $\mathrm{T}_{\text {reg }} \mathrm{s}$ is a major immune escape mechanism in cancer ${ }^{22}$ and increased tumor-infiltrating and/or circulating $\mathrm{T}_{\text {reg }} \mathrm{s}$ are correlated with detrimental outcomes ${ }^{23}$. Their role in colorectal cancer (CRC) remains unclear ${ }^{24,25,26,27}$, as $\mathrm{T}_{\text {reg }}$ s also suppress chronic inflammation, which is a cancer-promoting factor in inflammatory bowel disease (IBD)-associated $\mathrm{CRC}^{28,29}$. We described a sub-population of RORyt ${ }^{+} T_{\text {reg }}$ s that expands in the tumor and peripheral blood (PB) of CRC patients throughout disease progression ${ }^{30}$. While these cells still suppress $\mathrm{T}$ cell proliferation, they are also proinflammatory, as they express IL-17 and promote mast cell degranulation. In murine polyposis models, we causatively linked the pro-inflammatory skewing of $T$ cells to activation of Wnt/ $\beta$-catenin-signaling during thymic development ${ }^{31}$.

Here, we show that RORyt ${ }^{+} \mathrm{T}_{\text {reg }}$ s expressing the pro-inflammatory cytokines IL-17, IFNg, and TNFa expand progressivelly in both IBD and IBD/CRC patients and in mouse models of colitis and colitis/dysplasia. We establish that enhanced Wnt/ $\beta$-catenin signaling is responsible for the induction of pro-inflammatory $\mathrm{RORYt}^{+} \mathrm{T}_{\text {reg }}$ s. Moreover, we provide a molecular mechanism that explains this disease-associated phenomenon. We found that TCF-1 and Foxp3 co-bind regulatory elements, most importantly active enhancers, of genes that are involved in pro-inflammatory processes and limit their expression. Upon $W n t / \beta$-catenin activation, these genes gain newly accessible chromatin sites and become transcriptionally upregulated. Hence, the upregulation of $\beta$-catenin imposes a pro-inflammatory phenotype onto $\mathrm{T}_{\text {reg }}$ s by interfering with TCF-1/Foxp3-mediated gene suppression.

\section{Results}

Frequencies of $\beta$-catenin ${ }^{\text {high }}$ RORyt ${ }^{+} T_{\text {reg }}$ increase in the PB and colonic mucosa of IBD patients during inflammation and malignant disease progression. We previously established that RORyt ${ }^{+} \mathrm{T}_{\text {reg }} \mathrm{s}$ expand in sporadic CRC patients ${ }^{30}$. To understand the etiology of this expansion, we investigated IBD, which represents a unique platform to study the mechanisms underlying the emergence and distribution of $\mathrm{RORYt}^{+} \mathrm{T}_{\text {reg }} \mathrm{s}$ during inflammation and progression to $\mathrm{CRC}$. We analyzed colonic tissue and/or PB samples from 65 IBD patients, with or without dysplasia (IBD/Dys), and compared them to a group of 20 healthy donors (HD; Table S1). 
We first determined the frequencies of circulating fractions ( $F r$. $)$ of naïve $T_{\text {reg }} s\left(F r . I=C D 45 R A^{+} F o x p 3^{\text {int }}\right)$, activated $\mathrm{T}_{\text {reg }} \mathrm{s}\left(\mathrm{Fr}\right.$.ll=CD45RA-Foxp3 ${ }^{\text {high }}$ ), and activated conventional T cells (Fr.III= CD45RA-Foxp3 ${ }^{\text {int }}$ ) within $C D 4^{+} T$ cells as suggested by Sakaguchi and colleagues (Fig. 1a-e) ${ }^{32}$, 27 . Previously, we reported that Fr.ll of activated $\mathrm{T}_{\text {reg }} \mathrm{s}$ selectively increased in sporadic $\mathrm{CRC}$ compared to $\mathrm{HDs}^{30}$. Likewise, here we show a progressive enrichment of Fr.II $\mathrm{T}_{\text {reg }}$ frequencies from HDs to IBD and IBD/Dys (Fig. 1C). We also observed a progressive increase of non- $\mathrm{T}_{\text {reg }}$ Fr.III T cells, which were suggested to be prognostic for CRC clinical outcomes (Fig. 1C) ${ }^{27}$. We previously defined proinflammatory $T_{\text {reg }} s$ in patients by their expression of RORyt. We therefore assessed the proportions of RORyt-expressing cells within the Sakaguchi $T_{\text {reg }}$ fractions (Fig. 1b-d) as well as in the classically defined CD25 $5^{+}$Foxp $3^{+} \mathrm{CD} 127^{-} \mathrm{T}_{\text {reg }}$ population (Fig. S1a-c). In accordance with our previous work, RORyt ${ }^{+}$cells were enriched within Fr.ll of activated $\mathrm{T}_{\text {reg }} \mathrm{s}$ in IBD/Dys patients (Fig. 1d). Similarly, frequencies of RORyt ${ }^{+}$within $\mathrm{CD}^{2} 5^{\text {hi }}$ Foxp ${ }^{+} \mathrm{CD} 127^{-} \mathrm{T}_{\text {reg }}$ s also increased in IBD and IBD/Dys patients compared to HDs (Fig. S1b, c). Conclusively, PB RORYt ${ }^{+} \mathrm{T}_{\text {reg }}$ frequencies were elevated in IBD and IBD/Dys patients.

Previously, we causatively linked the skewing of $\mathrm{CD} 4^{+}$conventional T cells towards a Th17/proinflammatory phenotype to the activation of the Wnt/ $\beta$-catenin pathway ${ }^{31}$. Hence, we assessed the

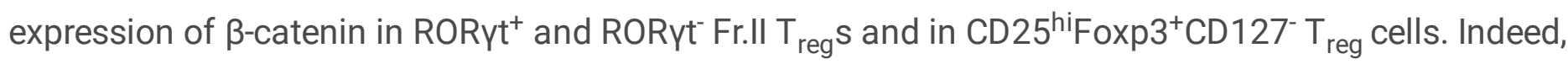
RORyt expression in $\mathrm{T}_{\text {reg }} \mathrm{S}$ uniformly correlated with enhanced $\beta$-catenin protein levels (Fig. 1b, e and Fig. S1d-e). Specifically, $\beta$-catenin expression was significantly higher in RORYt ${ }^{+}$compared to RORyt ${ }^{-}$Fr.II reg $_{\text {reg }}$ in patients and HDs (Fig. 1e). Further validating previous findings by our group and others ${ }^{21,30}$, the

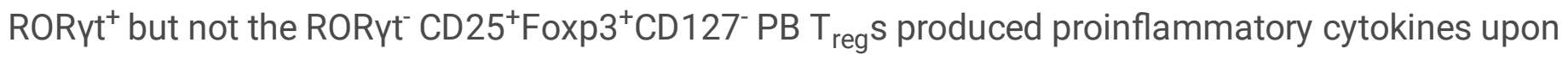
stimulation with PMA/lonomycin (Fig. 1f-i and Fig S1d-g). More precisely, RORyt ${ }^{+} \mathrm{PB} \mathrm{T}_{\text {reg }}$ s intracellularly accumulated IL-17, IFNg, and TNFa (Fig. $1 \mathrm{f}$-h) and a fraction of them were even found to be double producers for TNFa and IL-17 (Fig. 1i). Importantly, a significantly higher percentage of RORgt ${ }^{+} \mathrm{PB} \mathrm{T}_{\text {reg }} \mathrm{S}$ produced IFNg in IBD and IBD/Dys patients compared to HDs (Fig. 1g-h). Furthermore, compared to RORgt, co-expression of IL-17 and TNFa was significantly more frequent in RORgt ${ }^{+} \mathrm{PB} \mathrm{T}_{\text {reg }} \mathrm{S}$ of IBD and IBD/Dys patients but not of HDs (Fig. 1i). These findings show that chronic inflammation in IBD and IBD/Dys conincides with the increase in frequencies of the previously described RORgt ${ }^{+} T_{\text {reg }}$ population in the PB which produces multiple pro-inflammatory cytokines.

Next, we determined the frequencies of tissue-resident RORYt ${ }^{+} T_{\text {reg }} s$ in IBD patients by purifying mononuclear cells (MNCs) from inflamed (INF) and less inflamed ('margin', M) colonic mucosa samples. We found substantially higher frequencies of total Foxp $3^{+} \mathrm{T}$ cells $(\sim 30 \%)$ within tissue-resident $\mathrm{CD} 4^{+} \mathrm{T}$ cells compared to the PB. Moreover, tissue-resident total Foxp $3^{+} \mathrm{T}$ cells were significantly increased in IBD/Dys compared to IBD patients (Fig. 1j) for both $\mathrm{M}$ and INF areas. We also found that frequencies of RORyt ${ }^{+} \mathrm{T}_{\text {reg }} \mathrm{s}$ increased from the margin to the inflamed mucosa (Fig. 1k-I). Like their circulating counterparts, tissue-resident RORyt ${ }^{+} T_{\text {reg }}$ s expressed significantly higher levels of $\beta$-catenin compared to 
RORyt $^{-} T_{\text {reg }}$ s (Fig. 1m-n) and produced IL-17 upon stimulation (Fig. S1h, Fig. 10). Thus, circulating RORyt ${ }^{+}$ $\mathrm{T}_{\text {reg }} \mathrm{s}$ in IBD/Dys patients share major similarities with tissue-resident RORyt ${ }^{+} \mathrm{T}_{\text {reg }} \mathrm{s}$ and are likely tissue/tumor derived.

To explore possible RORyt ${ }^{+} \mathrm{T}_{\text {reg }}$ tumor infiltration we analyzed datasets from The Cancer Genome Atlas (TCGA, Research Network: https://www.cancer.gov/tcga) and determined how expression changes of Wnt/ $\beta$-catenin, $T_{\text {reg, }}$, and Th17 pathway genes were connected. To do this, we calculated the average zscores over all genes in the 'human WNT signature' (KEGG_human_WNT) ${ }^{33,34}$ and signatures containing genes that are transcriptionally up-regulated in Th17 cells (TH17_UP) and $T_{\text {reg }} s$ ( $\left.T_{\text {reg_- }} U P\right)$ that were kindly provided to us by Dr. Benoist and colleagues ${ }^{10}$. The TH17_UP positively correlated with the KEGG_human_WNT signature ( $p<0.001$, Spearman-score $r=0.5604$, red dots Fig. $1 p)$ and mirrored the equally strong positive correlation between the $T_{\text {reg_}} U P$ and the KEGG_human_WNT ( $p<0.001$, Spearmanscore $r=0.6161$, blue dots Fig. $1 p$ ) signatures. Also, the TH17_UP and $T_{\text {reg_}} U P$ signatures showed a strong positive correlation $(p<0.001$, Spearman-score $r=0.7836$, Fig. $S 1 i)$. This suggests that the $T_{\text {reg }}$ infiltrate in CRC tumors with an activated Wnt signature may possess Th17-like traits.

We further assessed whether the enhanced expression of $\mathrm{T}_{\text {reg }} / \mathrm{Th} 17$ signature genes could be correlated with adverse survival in the TCGA cohort, as the expression of Th17 associated genes was previously linked to detrimental outcome in $\mathrm{CRC}^{35}$. The effect on survival was tested with a machine learning approach. Coefficient values were derived from the machine learning approach for each gene in the TH17_UP, Treg_UP, and the combined TH17_UP/Treg_UP signatures via Cox proportionalhazards regression (example for the TH17_UP signature is shown in Fig. S1k,I) (Table S2). The genes whose enhanced expression predicted decreased survival included, amongst others, LEF-1 and MAF. We then divided patients, based on the median score of the TH17_UP, Treg_UP and combined TH17_UP/Treg_UP signature (TH17_UP signature shown as example in Fig. S1i). We interogated the survival outcomes of groups with above versus below the median score of the weighted signatures. These analyses indicated that above-median signature scores for the TH17_UP (Fig. 1q, p<0.0001, log-

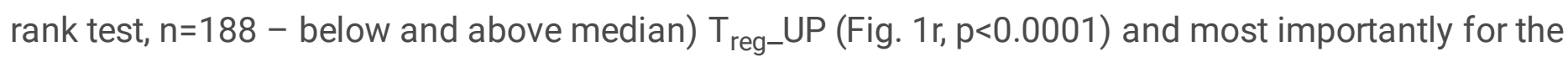

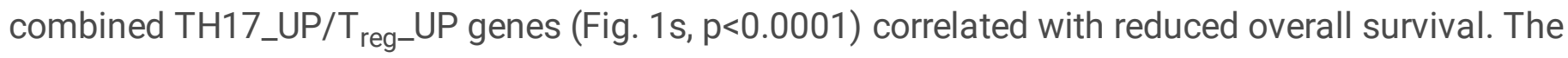
significant detriment in survival for the combined weighted signature (TH17_UP/Treg_UP) further supports the suggestion from the unweighted Spearman correlations that a $\mathrm{T}_{\text {reg }}$ tumor infiltrate with Th17-traits might results in adverse survival.

\section{Ex vivo stabilization of $\beta$-catenin in human $\mathrm{T}_{\text {reg }} s$ is sufficient to induce the pro-inflammatory phenotype.} Given our finding that RORyt expression in $\mathrm{T}_{\text {reg }} \mathrm{s}$ correlates with increased $\beta$-catenin levels in IBD(/Dys) patients, we investigated whether $\beta$-catenin stabilization was sufficient to induce RORyt and proinflammatory cytokine expression in HD $\mathrm{T}_{\text {reg }}$ s. Therefore, we treated HD PBMCs ex vivo with the GSK-3 $\beta$ inhibitor Chiron (CHIR99021) to stabilize $\beta$-catenin protein. Levels of $\beta$-catenin (Fig. 2a) and RORyt (Fig. 2c) were assessed in $\mathrm{CD}^{+} \mathrm{CD}_{2} 5^{+} \mathrm{Foxp} 3^{+} \mathrm{T}_{\text {reg }} \mathrm{s}$ after 4 (d4) and 7 (d7) days of culture with Chiron. 
Intracellular $\beta$-catenin (Fig. 2b) as well as RORyt (Fig. 2d) levels were significantly elevated in $T_{\text {reg }}$ after Chiron treatment at both time points. While $\beta$-catenin expression plateaued on $\mathrm{d} 4$, RORyt expression in Chiron-treated $\mathrm{T}_{\text {reg }} \mathrm{s}$ increased significantly between $\mathrm{d} 4$ and $\mathrm{d} 7$ (Fig. 2d).

In addition to $\beta$-catenin regulation, GSK-3 $\beta$ phosphorylates STAT proteins in T cells ${ }^{36,37}$, which promote the expression of pro-inflammatory cytokines IL-17 (STAT3) ${ }^{38,39}$ and IFN-y (STAT1) ${ }^{40,41}$. Hence, GSK-3 $\beta$ inhibition cannot be used to assess downstream cytokine production in these ex vivo cultures.

Alternatively, in human primary T cell cultures ${ }^{19}$ and in murine thymocytes ${ }^{42}$ it was shown that TCR engagement stabilizes $\beta$-catenin. To determine the physiological effect of $\beta$-catenin activation, we cultured HD PBMCs for $4 d$ in the presence of its natural activator, Wnt3a, in combination with CD3/CD28beads, CD3/CD28-beads alone, or vehicle control (Fig. 2e). CD3/CD28-beads were removed from cultures on $\mathrm{d} 4$ and Fr.II $T_{\text {reg }}$ cells (Fig. 2f) were analyzed for $\beta$-catenin and RORyt expression (Fig. 2g,h) as well as cytokine production (Fig. 2i,j) on d6. Intracellular levels of $\beta$-catenin and RORgt directly correlated (Fig $2 \mathrm{~g}$ ) and increased significantly in Wnt3a/CD3/CD28 and CD3/CD28 compared to vehicle control treated Fr.ll $\mathrm{T}_{\text {regs }}$ (Fig. 2h). Similarly, a significantly higher frequency of Wnt3a/CD3/CD28 and CD3/CD28 treated Fr.ll $\mathrm{T}_{\text {reg }}$ s expressed the proinflammatory cytokines IL-17, IFNy, and TNFa (Fig. 2j) after PMA/lonomycin stimulation. As observed in IBD(/Dys) patients, expression of IL-17 and TNFa (Fig. 2i,k) coincided and a smaller proportion of Fr.II $\mathrm{T}_{\text {reg }}$ s even co-expressed IL-17 and IFNy. Interestingly, gut homing receptor CCR9

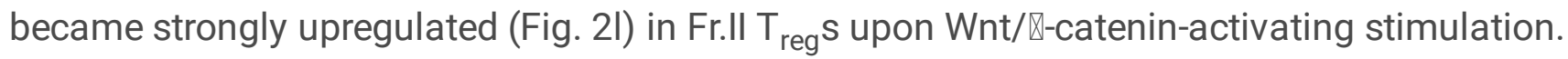

Conclusively, TCR stimulation in combination with activating Wnt-signals induced a pro-inflammatory phenotype in Fr.II $\mathrm{T}_{\text {reg }}$ s that is indentical with that observed in IBD(/Dys) patients (Fig. 1). Hence, these observations imply that $\beta$-catenin stabilization in primary human PBMCs is sufficient to drive the proinflammatory phenotype of $\mathrm{T}_{\text {reg }}$ s observed in IBD and CRC patients.

\section{Activated RORyt ${ }^{+}$sub-populations of $\mathrm{T}_{\text {reg }}$ s expressing gut homing receptors peripherally expand during disease progression in a murine IBD/CRC model. To recapitulate our findings in patients and further} analyze IBD/CRC-associated RORYt ${ }^{+} \mathrm{T}_{\text {reg }}$ s, we used our previously established murine APC ${ }^{\triangle 468}\left(\mathrm{APC}^{\Delta}\right)$ polyposis model ${ }^{43}$. In this model, translation of the adenomatous polyposis coli (APC) protein prematurely terminates due to ablation of exons 11 and 12, resulting in a truncated, nonfunctional 468 aa protein. The pathology of $\mathrm{APC}^{\Delta}$ resembles $\mathrm{APC}^{\mathrm{Min} /+}$ mice. They develop intestinal polyposis that spreads to the colon over time, and succumb to disease between 6-8 month of age $\mathrm{e}^{44,45}$. In previous work we used this model to demonstrate that $\mathrm{T}_{\text {reg }}$-specific ablation of Rorc reduced polyp burden in APC ${ }^{\Delta}$ mice ${ }^{30}$. Treatment of $\mathrm{APC}^{\mathrm{Min} /{ }^{+}}$mice with dextran sodium sulfate (DSS), which causes colitis in mice ${ }^{46}$, leads to the development of invasive $\mathrm{CRC}^{47,48}$. We established invasive colonic lesions (Fig. 3b), by treating 3-4month-old APC ${ }^{\Delta}$ mice with 3 rounds of $2 \%$ DSS in the drinking water for 7 days followed by 2 weeks recovery (Fig. 3a). This regimen led to colon shortening and a highly significant increase in colonic adenomas compared to untreated $\mathrm{APC}^{\Delta}$ mice (Fig. 3c). 
Next, we assessed the frequencies of total colonic $T_{\text {reg }} s$ (Fig. 3d) and RORyt ${ }^{+} T_{\text {reg }} s$ (Fig. 3e). Each of the three pathologic conditions colitis (WT+DSS), polyposis (APC $\left.{ }^{\Delta}-D S S\right)$, and IBD/CRC (APC $\left.{ }^{\Delta}+D S S\right)$ displayed increased colonic CD $25^{+}$Foxp $^{+} \mathrm{T}_{\text {reg }}$ (Fig. 3d) and RORyt ${ }^{+} \mathrm{T}_{\text {reg }}$ (Fig. 3e) frequencies compared to naïve mice (WT-DSS). As observed in IBD patients, $\beta$-catenin expression was significantly higher in RORyt ${ }^{+} T_{\text {reg }}$ s than in RORyt ${ }^{-} T_{\text {reg }} s$ for all treatment groups, (Fig. 3f). However, total $T_{\text {reg }} s$ (Fig. 3d) and RORYt $^{+} \mathrm{T}_{\text {reg }} \mathrm{s}$ (Fig. 3e) trended towards even higher frequencies in IBD/CRC compared to colitis or polyposis alone. We speculated that specific sub-populations of RORyt ${ }^{+} \mathrm{T}_{\text {reg }}$ s increased under IBD/CRC conditions, but the effect was masked by the overall increase of $T_{\text {reg }} \mathrm{s}$. Therefore, we designed two flow cytometric panels comprising CD4, Foxp3, CD25, RORyt, and $\beta$-catenin to distinguish distinct RORyt ${ }^{+}$and RORyt ${ }^{-} T_{\text {reg }}$ subsets. The first panel assessed tissue and inflammation homing markers; gut-homing receptor CCR9, inflammation-homing receptor CCR6, and tissue-residency marker CD103. The second panel focused on proliferation and activation markers Ki67, CD44, CD69, and CD62L (Fig. 3g). We gated on $\mathrm{CD}^{+}{ }^{+} \mathrm{CD} 25^{+} \mathrm{Foxp}^{+} \mathrm{T}_{\text {reg }}$ s from spleen (SPL) and colon MNCs, concatenated the populations from all experimental groups, and performed tSNE analysis. RORyt ${ }^{+} \mathrm{T}_{\text {reg }}$ gates were then superimposed onto the respective tSNE landscape revealing unique populations (Fig. S2a,b). These landscapes show that the complexity of RORyt ${ }^{+} \mathrm{T}_{\text {reg }}$ populations in the colon is greater than in the spleen (tissue and inflammation homing panel: colon=8, SPL=2 populations, activation panel: colon=8, $S P L=3$ populations, Fig $3 \mathrm{~g}$ ). To trace the origin of these cells, we focused on populations that expressed the same markers between the spleen and colon (red arrows, Fig. 3g). Analysis of expression profiles (Fig. S2a,b) revealed that RORyt ${ }^{+}$ $\mathrm{T}_{\text {reg }}$ populations shared between the colon and spleen expressed CCR9/CD103, and Ki67/CD44 (Fig. 3g). Cumulative analysis confirmed that $\mathrm{CCR}^{+} \mathrm{CD} 103^{+}$(Fig. 3h) and $\mathrm{CD} 44^{+} \mathrm{Ki}^{+} 7^{+}$(Fig. 3i) RORyt ${ }^{+} \mathrm{T}_{\text {reg }}$ frequencies in the IBD/CRC group (APC ${ }^{{ }+D S S}$ ) increased compared to the untreated WT, colitis (WT+DSS), and polyposis (APC ${ }^{\Delta}$-DSS) groups for colonic and splenic samples. Moreover, polyposis (APC ${ }^{\Delta}$-DSS) or colitis (WT+DSS) alone also showed elevated frequencies of the shared $T_{\text {reg }}$ populations in the colon, compared to the untreated group. This suggested that during IBD/CRC carcinogenesis a subpopulation of highly activated and proliferative RORyt ${ }^{+} \mathrm{T}_{\text {reg }} s$ with gut-homing properties expanded in the colon and the periphery (SPL). It further indicated diversity within the RORYt ${ }^{+} \mathrm{T}_{\text {reg }}$ subset, particularly in the colon.

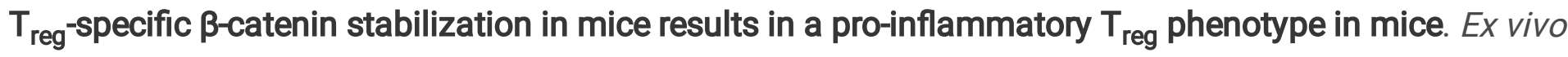
Wnt/ $\beta$-catenin pathway activation in primary human $\mathrm{HD} \mathrm{T}_{\text {reg }} \mathrm{s}$ recapitulated the phenotype of RORyt ${ }^{+}$ $\mathrm{T}_{\text {reg }}$ s observed in patients (Fig. 2). Accordingly, we examined in mice whether $\mathrm{T}_{\text {reg }}$-specific activation of the Wnt/ $\beta$-catenin pathway was sufficient to induce RORyt ${ }^{+} T_{\text {reg }}$ s. We introduced the $c t n n b 1^{f(\text { (ex3) }}$ allele ${ }^{49}$ into the Foxp3 $3^{\mathrm{YFP}-\mathrm{Cre}}$ mice established by Rudensky and colleagues ${ }^{50}$. Cre-excision of exon 3 encoding the $\beta$-catenin degradation domain leads to intracellular accumulation of $\beta$-catenin protein. 
As the Foxp3 gene resides on the $\mathrm{X}$-chromosome, male mice are hemizygous. Male Foxp3 ${ }^{\mathrm{YFP} \text {-Cre }}$ Ctnnb1 $^{\mathrm{fl}(\mathrm{ex} 3)}$ (CAT) mice showed severe X-linked, scurfy-like ${ }^{51,52}$ immune-pathology, (Fig. S3b) they were smaller than Foxp3 ${ }^{\text {YFP-Cre } W T ~(C r e) ~ l i t t e r m a t e s ~ a n d ~ d i e d ~ ~ 3-4 ~ w e e k s ~ a f t e r ~ b i r t h ~(F i g . ~ S 3 a) . ~ T h e y ~ f u r t h e r ~}$ presented enlarged secondary lymphoid organs, particularly peripheral lymph nodes (pLN), and splenomegaly (Fig. S3c). Histological assessment via H\&E staining of paraffin-embedded tissue sections revealed a reduced thymic cortex and severe immune infiltrates in lungs and liver (Fig. S3d, blue arrows). These observations mirrored those of a recent study using a different Foxp3Cre ${ }^{53}$ with the same floxed $\beta$ catenin allele ${ }^{21}$.

The observed pathology in CAT mice implied a lymphoproliferative disorder. Hence, we next assessed the activation status of conventional T cells in the peripheral lymphoid organs and thymi of 21-day-old mice. Compared to Cre-only, CAT mice had increased frequencies of total $\mathrm{CD}^{+}$cells in the spleen and pLNs; with a decrease of $C D 4^{+} T$ helper $\left(T_{H}\right)$ cells in the pLNs and $\mathrm{mLNs}$ and an increase of CD8 ${ }^{+}$cytotoxic $T$ cells (CTLs) in the spleen (Fig. S3e), indicating imbalanced T cell proliferation. This was further supported by the increase of $\mathrm{Ki}^{+} \mathrm{T}_{\mathrm{H}}$ cells and CTLs in CAT compared to WT mice (Fig S4a-b). Moreover, CD25-Foxp3 ${ }^{-} C D 4^{+} T_{H}$ cells and CTLs were strongly activated in CAT mice. The frequencies of $T_{H}$ cells and CTLs expressing the activation markers CD44, CD69, and CD25 were significantly increased in all peripheral lymphoid organs whereas the fractions of $T$ cells expressing the naïve $T$ cell marker $C D 62 \mathrm{~L}$ was reduced. (Fig. S4a-b).

The highly activated effector T cell compartment in CAT mice suggested that $\beta$-catenin stabilization altered $T_{\text {reg }}$ function. $T_{\text {reg }}$ frequencies strongly decrease within $\mathrm{mLNs}$ and spleens of CAT mice, however thymic $T_{\text {reg }}$ generation was unaltered (Fig. 4a, Fig. S4c). In peripheral $T_{\text {reg }}$ s, Foxp3 expression mildly increased (Fig. 4b), whereas expression of the $T_{\text {reg }}$ marker Neuropilin (Fig. S4d) did not change. Similar to the human ex vivo cultures, $\beta$-catenin stabilization (Fig. 4c) was sufficient to uniformely upregulate RORyt (Fig. 4d) levels in CAT ( $\beta$-catenin ${ }^{\text {high }}$ ) compared to $C r e T_{\text {reg }}$ s. Moreover, $\beta$-catenin ${ }^{\text {high }} \mathrm{T}_{\text {reg }} s$ were highly activated, as evidenced by increased frequencies of CD44 ${ }^{+}$(Fig. 4e) and CD69 ${ }^{+}$(Fig. 4f) $\mathrm{T}_{\text {reg }} \mathrm{S}$ and decreased CD62 $\mathrm{L}^{+} \mathrm{T}_{\text {reg }} \mathrm{s}$ (Fig. 4g) in CAT mice. Likewise, $\mathrm{T}_{\text {reg }} \mathrm{s}$ in CAT mice were more proliferative, demonstrated by Ki67 staining (Fig. 4h). Lastly, we tested the ability of $\beta$-catenin ${ }^{\text {high }} \mathrm{T}_{\text {reg }}$ s to suppress proliferation of polyclonally activated, CFSE-labeled CD $4^{+} \mathrm{CD} 25^{-} \mathrm{T}$ effector cells $\left(\mathrm{T}_{\text {eff }}\right)$ in vitro. Compared to control Cre $T_{\text {reg }} s, \beta$-catenin ${ }^{\text {high }} T_{\text {reg }}$ s had significantly reduced suppressive function for all $T_{\text {reg }}: T_{\text {eff }}$ ratios tested (Fig. 4i).

Conclusively, $\beta$-catenin stabilization in murine $T_{\text {reg }} s$ was sufficient to up-regulate RORyt and induce an activated, pro-inflammatory phenotype that mirrored the systemically expanding RORyt ${ }^{+} \mathrm{T}_{\text {reg }}$ subpopulation observed in the $\mathrm{APC}^{\triangle}$ /DSS model (Fig. 3i). The lymphoproliferative disease in CAT mice could thus be attributed to a combination of the activated phenotype and the reduced suppressive function of $\beta$-catenin ${ }^{\text {high }} \mathrm{T}_{\text {reg }} \mathrm{s}$. 
$\beta$-catenin ${ }^{\text {high }} T_{\text {reg }} s$ are competitively disadvantaged in a chimeric setting and spontaneously express proinflammatory cytokines. Foxp3 ${ }^{\text {YFP-Cre }}$ heterozygous females represent natural chimeras of $\beta$ catenin ${ }^{\text {high }} /$ YFP $^{+}$versus WT/YFP ${ }^{-} \mathrm{T}_{\text {reg }}$ s due to random X-chromosome inactivation. These mice provide the opportunity to test the pathogenic potential of $\beta$-catenin ${ }^{\text {high }} /$ RORythigh $T_{\text {reg }} s$ in a competitive chimeric setting. Interestingly, compared to hemizygous males, heterozygous Foxp $3^{\mathrm{Cre}(+/)}$ females that carry the Ctnnb1 $1^{\mathrm{fl}(\mathrm{ex} 3)}$ allele are healthy.

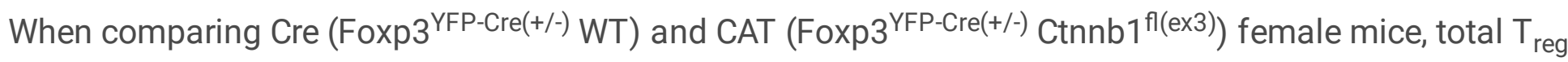
numbers in the spleen and thymus were identical between genotypes (Fig. 5a). The frequency of YFP+ $\mathrm{T}_{\text {reg }} \mathrm{S}$, however, was drastically reduced in CAT compared to Cre females (Fig. 5b). Cumulative analysis of $\mathrm{YFP}^{+}$and $\mathrm{YFP}^{-} \mathrm{T}_{\text {reg }}$ fractions showed that $\mathrm{YFP}^{+} \mathrm{T}_{\text {reg }}$ s only accounted for $1-3 \%$ of total $\mathrm{T}_{\text {reg }} \mathrm{s}$ in all peripheral lymphoid organs of female CAT mice (Fig. 5c). The thymic output of $\mathrm{YFP}^{+} \mathrm{T}_{\text {reg }} \mathrm{s}$ was also reduced to $20 \%$ in CAT females. In Cre females the ratio of YFP $^{+} /$YFP $^{-} T_{\text {reg }} S$ was also slightly reduced to $40 \% / 60 \%$ (Fig. 5b,c). This could be attributed to the YFP-Cre transgene rendering Foxp3 expression in $\mathrm{YFP}^{+} \mathrm{T}_{\text {reg }}$ s mildly hypomorphic compared to $\mathrm{T}_{\text {reg }}$ s with an unaltered Foxp3 locus ${ }^{54}$. The persisting $\mathrm{YFP}^{+} /$ $\beta$-catenin ${ }^{\text {high }} \mathrm{T}_{\text {reg }}$ s had elevated RORyt expression (Fig. 5d) and an activated phenotype, as evidenced by increased CD44 expression (Fig. 5e) compared to CAT YFP- $\mathrm{T}_{\text {reg }}$ s but also compared to Cre $\mathrm{YFP}^{+} \mathrm{YFP}^{-}$ $\mathrm{T}_{\text {reg }}$ populations. In accordance to our findings in patients' RORgt ${ }^{+} \mathrm{T}_{\text {reg }} \mathrm{s}$, CAT YFP ${ }^{+} / \beta$-catenin ${ }^{\text {high }}$ compared to $\mathrm{YFP}^{-}$and to $\mathrm{Cre} \mathrm{YFP}^{+} / \mathrm{YFP}^{-} \mathrm{T}_{\text {reg }}$ populations spontaneously expressed proinflammatory cytokines IL-17, IFNg, and TNFa. Further mirroring our finding in patients (Fig. 1), a significant proportion of these $\mathrm{YFP}^{+} / \beta$-catenin ${ }^{\text {high }} \mathrm{T}_{\text {reg }}$ s co-expressed IL-17 and IFNg or IL-17 and TNFa (Fig. 5f-i). In accordance to findings in human samples, the gut-homing receptor CCR9 was upregulated (Fig. S5a) as shown for wnt3a/anti-CD3/28-treated human $\mathrm{T}_{\text {reg }} \mathrm{s}$ (Fig. 2l). Conclusively, in a non-inflammed setting, $\beta$ catenin $^{\text {high }}$ RORyt ${ }^{\text {high }} \mathrm{T}_{\text {reg }}$ s have a competitive disadvantage in chimeric mice. Although they do not spontaneously cause disease, they are poly-functional in producing proinflammatory cytokines.

\section{The DNA-binding partner of $\beta$-catenin, TCF-1, and Foxp3 co-bind accessible chromatin at gene loci that} are crucial for $\mathrm{T}_{\text {reg }}$ function. Previous work indicated that $\beta$-catenin ${ }^{20} /$ TCF- $1^{13}$ occupy DNA together with Foxp3. Thus, we anticipated that precise mapping of TCF-1 and Foxp3 co-binding in $T_{\text {reg }}$ s, could provide a molecular explanation for the change in the phenotype of $T_{\text {reg }} s$ that overexpress $\beta$-catenin. To address this, we analyzed TCF-1 DNA binding in $\mathrm{T}_{\text {reg }}$ s through chromatin immunoprecipitation and deep sequencing (ChIPseq) in Foxp3 ${ }^{\text {YFP-Cre }}$ WT $\mathrm{T}_{\text {reg }}$ s. Furthermore, we assessed regions of accessible chromatin in $\mathrm{T}_{\text {reg }} \mathrm{s}$ via transposase-accessible chromatin approach and deep sequencing (ATAC-seq). We also utilized available data ${ }^{55}$ for Foxp3-binding (Foxp3-ChIPseq), histone marks including; monomethylated histone 3 at Lys4 (H3K4me1), tri-methylated H3 Lys4 (H3K4me3), acetylated H3 at Lys27 (K3K27Ac), trimethylated H3 at Lys27 (H3K27me3), and Methyl-CpG binding domain-based capture and sequencing (MBD-seq). 
We assigned TCF-1 (Fig. S6a-C) and Foxp3 (Fig. S7a-C) ChIPseq-peaks to specific gene regulatory regions by performing $K$-means clustering guided by the surrounding chromatin modifications. $14.6 \%$ of TCF-1 (Fig. S6a) and $24.0 \%$ of Foxp3 (Fig. S7a) binding sites, were assigned to active enhancers (AE, enriched for H3K4me1, H3K27Ac), $41.6 \%$ and $39.3 \%$ of transcription factor binding sites to poised enhancers (PE, enriched for $\mathrm{H} 3 \mathrm{~K} 4 \mathrm{me} 1$, lacking $\mathrm{H} 3 \mathrm{~K} 27 \mathrm{Ac}$ ), and $44.0 \%$ and $36.7 \%$ to promoters (Pr, enriched for $\mathrm{H} 3 \mathrm{~K} 4 \mathrm{me}$, H3K27ac, and accessible chromatin (ATAC-seq signal)). Both TCF-1 and Foxp3 preferentially bound open and poised chromatin as their binding sites rarely overlapped with the repressive histone mark H3K27me3 (Fig. S6b-c, Fig. S7b-C). We next performed motif analysis for TCF-1- and Foxp3-bound sites. TCF-1-bound enhancer sites were enriched for the TCF-1 consensus motif, while both promoter and enhancer Foxp3-bound sites were enriched for the Foxp3 motif (Fig S6d, Fig. S7d). The high enrichment for motifs of other factors like Ets, RUNX and YY family members agreed with previous evidence that TCF- $1^{14}$ and Foxp $3^{12}$ act in multi-molecular complexes. Pathway enrichment analysis (http://www.metascape.org) revealed that TCF-1 (Fig. S6e) and Foxp3 (Fig. S7e) binding to promoter and enhancer sites marked genes involved in $\mathrm{T}$ cell activation and other $\mathrm{T}$ cell processes, as well as in general DNA and RNA metabolism processes.

We further analyzed directly overlapping TCF-1 and Foxp3 co-bound sites (Fig. 6), which included 504 AE, 389 PE, and 1101 Pr (Fig. 6a). Examples for TCF-1 and Foxp3 co-bound gene loci at AE (Tcf 7), PE (Ccr7), and $\operatorname{Pr}$ (Stat1) sites are visualized as IGB tracks (Fig. 6f). The TCF-1 consensus motif, but not the Foxp3 binding motif, was highly enriched in the overlapping AE and Pr sites (Fig. 6d). Pathway enrichment analysis revealed that co-binding of TCF-1 and Foxp3, particularly at AE sites, marked genes involved in Th17 differentiation, T cell activation, and cytokine production pathways (Fig. 6e). Thus, in $\mathrm{T}_{\text {reg }}$ S TCF-1 and Foxp3 together occupied genes whose upregulation could explain the phenotype of RORyt ${ }^{+} \mathrm{T}_{\text {reg }} \mathrm{S}$ observed in IBD/CRC patients and mouse models.

\section{$\beta$-catenin stabilization drives the proinflammatory phenotype of RORyt ${ }^{+} \mathrm{T}_{\text {reg }}$ s through epigenetic and} transcriptional regulation of critical Foxp3 and TCF-1 co-bound genes. The classical Wnt signaling model posits that TCF-1 acts as a transcriptional repressor that becomes an activator once $\beta$-catenin binds and mediates epigenetic changes. Hence, one could speculate that, in a Wnt-off state, TCF-1 is part of Foxp3 repressor complexes ${ }^{12}$, which act together to limit the expression of genes that should not be permanently expressed in bona fide $\mathrm{T}_{\text {reg }} \mathrm{s}$. Thus, we postulated that $\beta$-catenin stabilization specifically affects the transcription and epigenetic states of these TCF-1/Foxp3 co-bound genes.

To explore this possibility, we performed ATAC-seq analysis of $\mathrm{YFP}^{+} \mathrm{CD} 25^{+} \beta$-catenin ${ }^{\text {high }} \mathrm{T}_{\text {reg }}$ s FACS sorted from CAT (Foxp3 ${ }^{\text {YFP-Cre }}$ Ctnnb $1^{\text {fl(ex3)}}$ ) and WT (Foxp3 ${ }^{\text {YFP-Cre }}$ ) mice (Fig. 7a). $\beta$-catenin ${ }^{\text {high }} \mathrm{T}_{\text {reg }} \mathrm{S}$ (23,084 peaks) had fewer accessible sites than $\mathrm{Cre}_{\mathrm{reg}} \mathrm{s}(29,566$ peaks). However, the sites that lost accessibility from Cre to $\beta$-catenin ${ }^{\text {high }}$ (Cre unique sites, 9831 peaks), already had low accessibility in Cre $T_{\text {reg }}$ s and yet were not fully closed in $\beta$-catenin ${ }^{\text {high }} T_{\text {reg }}$ s. The majority of sites remained consistently open for both genotypes (common, 19735 peaks) and 3349 sites gained de novo accessibility in $\beta$-catenin ${ }^{\text {high }}$ 


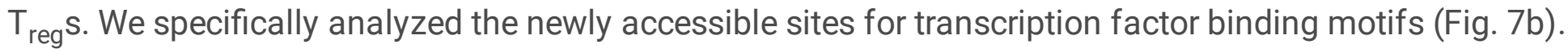
One of the most strongly enriched motifs was that of TCF-1 (Tcf 7), indicating that $\beta$-catenin together with TCF-1 may directly alter the accessibility of corresponding genes. Additionally, motifs of transcription factors that are involved in Th17 differentiation (Maf, RARg) and T cell activation (JunB) were highly enriched. The 3349 newly accessible sites corresponded to 2582 genes (Fig. 7a) of which 370 were cobound by TCF-1 and Foxp3 (Fig. 7c). Pathway enrichment analysis of these 370 genes revealed that T cell activation, cytokine production, and Th17 differentiation pathways were most significantly enriched (Fig. 7d).

We next performed RNA-seq expression profiling to investigate how the changes in chromatin accessibility impacted transcription in $\beta$-catenin ${ }^{\text {high }} \mathrm{T}_{\text {reg }}$ s. Differential expression testing yielded 3190 upand 2795 down-regulated (q-value $\leq 0.05$ ) genes in $\beta$-catenin ${ }^{\text {high }}$ compared to $C r e T_{\text {reg }}$. The upregulated list comprised genes that are essential for $T_{\text {reg }}$ function, including CTLA-4 and II2ra (CD25, Fig. 7e). Pathway enrichment analysis for significantly up-regulated genes that were co-bound by TCF-1 and Foxp3 (Fig. 7f-g) revealed an enrichment for the very same pathways identified by the newly accessible chromatin sites, namely, T cell activation and Th17 differentiation (Fig. S8).

We next assessed the dynamic chromatin accessibility changes following $\beta$-catenin stabilization of genes co-bound by TCF-1/Foxp3 in different regulatory regions. Therefore, we compared the $\log _{2}$-fold accessibility changes in $\beta$-catenin ${ }^{\text {high }} T_{\text {reg }}$ s over Cre $T_{\text {reg }}$ s for promoter, $P E$, and $A E$ co-bound to that of all differentially accessible genes (Fig. 8a). Although, genes co-bound by TCF-1/Foxp3 always gained accessibility, the AE-bound genes showed the strongest increase in accessibility in $\beta$-catenin ${ }^{\text {high }} \mathrm{T}_{\text {reg }}$ s (Fig. 8a). As AE-bound genes are most affected by $\beta$-catenin stabilization, we specifically searched for those that were up-regulated, gained accessibility, and were TCF-1/Foxp3 co-bound at AE sites in $T_{\text {reg }} S$ (Fig. 8b). This yielded in 49 genes, that were identified to belong to T cell activation and Th17 differentiation pathways (Fig. 8b). We further validated these findings via an inverse, non-supervised approach. We retrieved gene lists for the Th17 differentiation (102 genes) pathway (GSEA/MSigDB database), and used the same $\mathrm{T}_{\text {reg_ }}$ UP signature (195 genes) used for TCGA screening. The leukocyte migration (242 genes) pathway (GSEA/MSigDB database) was also assessed due to the observed increase in CCR9 expression in RORyt ${ }^{+} \mathrm{T}_{\text {reg }}$ s in humans (Fig. 2l) and mice (Fig. 3, Fig. S5d) suggesting their migration from the colon to the periphery. Comparing accessibility of genes in these pathways showed strong de novo accessible sites that arise after $\beta$-catenin activation in $\mathrm{T}_{\text {reg }} \mathrm{s}$ (red lines) compared to common (black lines) and WT sites (green lines, Fig. 8c). Moreover, gene set enrichment analysis $\left(\right.$ GSEA $^{56}$ ) showed a significant positive upregulation for Th17 differentiation and leukocyte migration pathways (Fig. 8d, Fig. S8). The $T_{\text {reg_ }}$ UP signature, however, was not consistently changed between WT and $\beta$-catenin ${ }^{\text {high }} T_{\text {reg }} s$ (Fig. 8d). This indicated that the core $T_{\text {reg }}$ program was not drastically altered, but non- $T_{\text {reg }}$ /effector $T$ cell functions were acquired upon $\beta$-catenin activation. For instance, activation of $\beta$ catenin led to the acquisition of a newly accessible site in the IFNy locus (Fig. 5e, black arrow), which may account for the ability of $\beta$-catenin ${ }^{\text {high }} T_{\text {reg }}$ s to express IFNy. Similarly, the IL-17 locus gained 
accessibility (Fig. 8e), which may account for spontaneous IL-17 production in $\beta$-catenin ${ }^{\text {high }} \mathrm{T}_{\text {reg }}$ s (Fig $5 h, i)$.

Overall, our findings indicated that $\beta$-catenin activation caused epigenetic and transcriptional changes of crucial Foxp3/TCF-1 co-regulated genes. This resulted in the induction of transcriptional programs responsible for Th17 differentiation/T cell activation that were super-imposed onto the $T_{\text {reg }}$ program and potentially conferred the observed pro-inflammatory phenotype to $\beta$-catenin ${ }^{\text {high }} \mathrm{T}_{\text {reg }}$ s.

\section{Discussion}

Diverse $T_{\text {reg }}$ populations that express transcriptional programs in addition to the core $T_{\text {reg }}$ signature as functional adaptation to the tissue microenvironment and inflammatory milieu have been described $1,2,3$, $4,5,6,7$. Here we address mechanisms that potentially underlie $T_{\text {reg }}$ functional changes in chronic inflammation and cancer, focusing on a population of RORYt ${ }^{+} T_{\text {reg }} s$ in the context of IBD and CRC. Our results show that during IBD and progression to dysplasia, RORyt-expressing $\mathrm{T}_{\text {reg }} \mathrm{S}$ that produce proinflammatory cytokines selectively expand in the colon and PB of patients. We linked this RORYt ${ }^{+} \mathrm{T}_{\text {reg }}$ phenotype to enhanced $\mathrm{Wnt} / \beta$-catenin signaling in both humans and animal models. Our molecular analyses provide evidence that TCF-1, the DNA-binding partner of $\beta$-catenin, together with Foxp3, controls the expression of genes involved in T cell activation and Th17-proinflammatory processes. The stabilization of $\beta$-catenin upregulates the expression of these genes by promoting chromatin accessibility and thereby superimposes pro-inflammatory traits onto the core $\mathrm{T}_{\text {reg }}$ program.

In healthy mice, a specific consortium of bacteria induces RORyt ${ }^{+} \mathrm{T}_{\text {reg }} \mathrm{s}$ mainly in the colon, where they regulate local inflammatory responses ${ }^{6,7}$. During IBD and progression to cancer, antigens from bacteria and from the chronically-inflamed, damaged mucosal tissue mediate persistent TCR-stimulation ${ }^{57}$. Furthermore, CRC tumors and the inflamed mucosa were shown to secrete enhanced levels of activating Wnt proteins ${ }^{58,59}$. This persistent TCR- stimulation ${ }^{19,42}$ in a Wnt rich environment has the potential to strongly activate Wnt/ $\beta$-catenin in T cells. Furthermore, mucosal barrier dysfunction in IBD and IBD/Dys likely enables the PB dissemination of the RORyt ${ }^{+} T_{\text {reg }} s$ in an antigen-driven manner leading to their systemic occurance. Indeed, our ex vivo experiments in human PBMCs indicate that TCR-stimulation and activating Wnt signals stabilize $\beta$-catenin in $T_{\text {reg }}$ s and increase the frequencies of RORyt ${ }^{+} T_{\text {reg }} s$, which express the pro-inflammatory cytokines IL-17, IFNy, and TNFa. A certain proportion of these RORyt ${ }^{+} \mathrm{T}_{\text {reg }} \mathrm{S}$ showed a poly-functional cytokine profile by co-expressing IL-17 and IFNg or IL-17 and TNFa simultaneously. Furthermore, genetic $\beta$-catenin stabilization in our Foxp3 ${ }^{\mathrm{Cre}}$-driven mouse model induces $\mathrm{T}_{\text {reg }}$ s that are homogenously RORyt ${ }^{\text {high }}$ and spontaneously produce IL-17, IFNY, and TNFa even in the absence of overt inflammation in a competitive chimeric setting. These findings suggest that constitutive Wnt/ $\beta$-catenin signaling in human as well as murine $T_{\text {reg }} s$ is sufficient to promote the pro-inflammatory RORyt $^{+} \mathrm{T}_{\text {reg }}$-phenotype. 
We previously showed increased frequencies of circulating RORYt ${ }^{+} \mathrm{T}_{\text {reg }} \mathrm{s}$ in patients with sporadic $\mathrm{CRC}^{30}$. Here we found that in IBD RORyt ${ }^{+} \mathrm{T}_{\text {reg }}$ s also expand systemically and have proinflammatory properties, promoting the notion that colonic and circulating RORYt ${ }^{+} \mathrm{T}_{\text {reg }} s$ may be related. This is supported by several findings. First the observation that $\beta$-catenin stabilization both in human PBMCs and in the chimeric mouse model upregulates gut-homing receptor CCR9. Second, the finding that the expanding RORYt $^{+} \mathrm{T}_{\text {reg }} \mathrm{s}$ in the IBD/CRC mouse model express CCR9 as well as tissue-residency marker CD103 undeline their colonic/tissue origin. Finally, the observation that $\beta$-catenin stabilization transcriptionally upregulates leukocyte migration genes including CCR7, which promotes cell entry to lymphoid tissues ${ }^{60}$, suggests altered migration. As mentioned above, mucosal barrier dysfunction ${ }^{61,62}$ can cause systemic microbial and tissue-specific antigen-spread during IBD/CRC and contribute to systemic immune dysregulation ${ }^{57}$. It was previously reported that $T_{\text {reg }}$ migration in breast cancer can be Ag-specific ${ }^{63,64}$. Similarly, the above conditions may facilitate migration of gut-tissue-resident RORyt ${ }^{+} \mathrm{T}_{\text {reg }} \mathrm{s}$ and/or support their maintenance in the periphery, thereby increasing their frequencies in IBD and CRC.

The epigenetic and transcriptional studies presented here focus on determining the mechanisms by which $T_{\text {reg }}$-specific $\beta$-catenin-activation promotes the disease-associated RORYt ${ }^{+} T_{\text {reg }}$ phenotype. In line with previous evidence that TCF- 1 interacted with the signature $T_{\text {reg }}$ transcription factor Foxp ${ }^{20}$, here we show that the genome-wide TCF-1-DNA binding in $\mathrm{T}_{\text {reg }}$ s substantially overlaps with that of Foxp $3^{13}$. Importantly, we find that TCF-1 and Foxp3 co-bind active enhancer regions of genes responsible for Th17 differentiation, $T$ cell activation, and leukocyte migration. Recent studies have shown that Foxp3 binding to active enhancers ${ }^{9}$ can activate or suppress expression of the associated genes depending on local interactions with other specific cofactors. In particular, Foxp3 likely acts as a repressor in complexes with YY1, EZH2, and Ikzf3 ${ }^{12}$. Based on current evidence ${ }^{20,13}$ and our own results, TCF-1 possibly participates in repressive regulatory complexes with Foxp3. The finding that the conserved YY1 binding motif is strongly enriched in the Foxp3/TCF-1 co-bound DNA-sites further supports this suggestion. Moreover, our findings that $\beta$-catenin stabilization enhances chromatin accessibility and the expression of certain TCF1/Foxp3 co-bound genes indicates that the TCF-1/Foxp3-mediated regulation of these genes depends on $\beta$-catenin expression levels. Enhanced $\beta$-catenin binding to TCF-1 may alter the TCF-1/Foxp3 regulation of these gene loci by displacing co-repressors to promote transcription. Indeed, the regions that gain de novo accessibility with $\beta$-catenin stabilization are enriched for the TCF-1 binding motif, suggesting that the $\beta$-catenin-mediated epigenetic changes involve TCF-1. These molecular changes mediated by $\beta$ catenin promote a duality in the phenotype of RORyt ${ }^{+} \mathrm{T}_{\text {reg }}$ s by enhancing pro-inflammatory Th17 differentiation and leukocyte migration pathways without abrogating the regulatory core $\mathrm{T}_{\text {reg }}$ signature.

In conclusion, our study establishes the progressive and systemic expansion of RORyt ${ }^{+} \mathrm{T}_{\text {reg }}$ S during IBD and progression to dysplasia and highlights activation of $\beta$-catenin as a major underlying molecular process. We show that TCF-1 and Foxp3 function together to control the expression of a proinflammatory program through binding to active enhancer regions of the associated genes. We further 
define that this regulation is driven by $\beta$-catenin stabilization, which promotes chromatin accessibility and enhances transcription of the TCF-1/Foxp3 co-regulated genes. $\beta$-catenin mediates upregulation of T cell activation and Th17 signature genes and superimposes this activated proinflammatory phenotype onto the core $T_{\text {reg }}$ program. Future studies should establish the ontology and TCR specificities of the diseaseassociated RORyt ${ }^{+} T_{\text {reg }} \mathrm{S}$, as well as their roles in perpetuating inflammation. It is curcial to understand the exact contributions of RORyt ${ }^{+} T_{\text {reg }}$ s to chronic intestinal inflammation and malignancies, which first and foremost should address the effects of pro-inflammatory cytokine production by these cells on disease progression.

The present data connects to our previous report that frequencies of pro-inflammatory $T_{\text {reg }} s$ increase in the blood of CRC patients ${ }^{30}$ and expands it to a pre-malignant setting of chronic inflammation. The step wise increase in the frequencies of RORyt ${ }^{+} T_{\text {reg }}$ s expressing IL-17, IFN- $y$, and TNFa from IBD to early dysplasia and CRC emphasizes the involvement of these cells in pro-inflammatory immune dysregulation fostering chronic inflammation and therewith acting cancer-promoting. Early intervention in cancer treatment is greatly needed. In this context frequencies of circulating RORyt ${ }^{+} T_{\text {reg }} \mathrm{s}$ could serve as a biomarker in IBD and CRC. Furthermore, our detailed molecular analysis of genes and processes affected

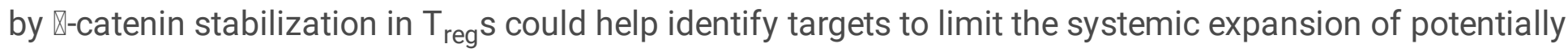
disease-promoting pro-inflammatory $\mathrm{T}_{\text {reg }} \mathrm{s}$ during $\mathrm{CRC}$.

\section{Materials And Methods}

Patients and Healthy Donors. 43 patients undergoing major surgery for Crohn's disease (CD)/ulcerative colitis (UC) treatment (proctocolectomy, colectomy, partial ileectomy, or combinations of the aforementioned procedures) were consented to donate $45 \mathrm{ml}$ of peripheral blood either directly preop or directly postop. Up to $4 \mathrm{~g}$ of fresh intestinal mucosal tissue was collected from inflamed, margin (in most cases less inflamed), and dysplastic areas from the surgical specimens (if made available for research purposes by pathology) from the same patients. Additionally, 22 IBD patients undergoing routine colonoscopy check-up visits and 20 healthy individuals participated to the study by donating up to $45 \mathrm{ml}$ of peripheral blood. Informed consent was obtained from all patients and healthy donors. The protocol was approved by the University of Chicago Division of the Biological Sciences Institutional Review Board (BSD IRB) and was performed in accordance to Federal Law.

\section{Isolation and flow cytometric analysis of PBMCs and MNCs from patient and healthy donor samples.}

PBMCs were isolated from peripheral blood samples using LymphoprepTM (Serumwerk Bernburg AG for Alere Technologies AS, via Stemcell 7851) density gradient centrifugation. MNCs were isolated from mucosal tissue samples using the Tumor Dissociation Kit, human (Miltenyi 130-059-929) and gentleMACS C Tubes (Miltenyi 130-093-237) following the instructions from the Lamina Propria Dissociation Kit, mouse (Miltenyi 130-097-410). MNCs isolated from blood and intestinal mucosa were either first re-stimulated with Cell Stimulation Cocktail plus protein transport inhibitors 500x (eBioscience 00-4975-93) for three hours in X-Vivo-20 media (LONZA 04-448-Q) or used directly for flow cytometric 
staining. Cells were stained for viability using the LIVE/DEAD Aqua fluorescent reactive dye (Molecular Probes - life technologies L34963), followed by surface staining with varying combinations of the following antibodies: CD3 (clone OKT3, BioLegend; UCHT1, ThermoFisher), CD4 (clone RPA-T4, BD), CD8 (RPA-T8, eBioscience/ThermoFisher Scientific/BD), CD25 (clone M-A251, BD), CD45RA (clone HI100, BD/BioLegend), CD127 (clone HIL-7R-M21, BD), CD279/PD-1 (clone EH12.1.H7, BioLegend), CD152/CTLA-4 (clone 14D3, eBioscience/ThermoFisher Scientific), CCR9 (L053E8, BioLegend (V27-580, $\mathrm{BD})$ and respective manufacturer isotype controls. Following surface staining, cells were fixed and permeabilized using Foxp3/Transcription Factor Fixation/Permeabilization Concentrate and Diluent (Affymetrix/eBioscience/ThermoFisher Scientific, 00-5521) and intracellular staining was performed for Foxp3 (clone 236A/E7, eBioscience/Thermo Fisher Scientific), RORyt (clone Q21-559, BD), $\beta$-Catenin (clone 14/Beta-Catenin, BD) IL-17 (clone BL 168, BioLegend), IFNy (clone 4S.B3, eBioscience/Thermo Fisher Scientific), TNFa (Mab11, BioLegend), Ki67 (Ki-67, BioLegend), and respective manufacturer isotype control antibodies. Samples were then acquired using a 4 laser/15 detector LSR-Fortessa instrument (BD) or an 5-Laser Aurora (Cytek ${ }^{\mathrm{TM}}$ ) instrument. For the spectral flow stainings Brilliant Stain Buffer Plus (BD) was added to the staining mixes according to manufacturer's insctructions. Isolated PBMCs were either analyzed directly ex vivo or frozen (10\% DMSO (SIGMA D2650), 90\% human AB Serum (Gemini 100-512)) and analyzed in batch directly after thawing.

Analysis of TCGA data. Spearman Correlations; The results are based on data generated by the TCGA Research Network: https://www.cancer.gov/tcga. Average z-scores for each individual gene in each of the signatures and for the tumors of each individual patient in the TCGA database were downloaded. Average z-scores are calculated as relative mRNA expression value of an individual gene and tumor, normalized to the gene's expression distribution in a reference population (defined as all samples that are haploid for the respective gene). Then, the z-scores of all genes in each individual signature were averaged over the number of genes in the respective signature, yielding average z-scores for the Th17_UP, Treg_UP, and KEGG_human_WNT signatures for each patient. Regularized Regression of TCGA Gene Expression; In order to determine the ability of the TH17 gene signature to inform patient-level survival, we deployed Cox proportional-hazards regression on colorectal cancer clinical data extracted from TCGA. We used Ridge regression regularization ${ }^{65}$ and chose an optimal penalty coefficient $\lambda$ using $10-$ fold cross validation optimized for partial likelihood deviance. The TH17 gene-based score for each patient was generated by multiplying the coefficient vector by the patient-level gene expression. Patient scores were dichotomized at the median and Kaplan-Meier curves were plotted. Statistical coding was performed using R version 3.1.2, and packages survival ${ }^{66}$, glmnet ${ }^{67}$, and survminer ${ }^{68}$.

In vitro Wnt/ $\beta$-catenin pathway activation in HD PBMCs. PBMCs were isolated via density gradient centrifugation under sterile conditions. Cells were adjusted to $1 \times 10^{7}$ cells in X-Vivo ${ }^{T M} 20$ media (LONZA, 04-448Q) supplemented with 10\% AB-Serum (Gemini, 100-512), $100 \mathrm{U} / \mathrm{ml}$ recombinant human (rh)-IL-2 (PreproTech, 200-02), 60 U/ml IL-4 (PreproTech, 200-04), and $1 \mathrm{ng} / \mathrm{ml}$ TGF- $\beta 1$ (PreproTech, 100-21) and seeded in 12-well plates at $2 \times 10^{6}$ cells/well. For the GSK3- $\beta$ inhibition, cells were treated with $6 \mu \mathrm{M}$ Chiron (CHIR99021, Sigma, SML1046) or DMSO vehicle control for 6 days total, supplemented with $3 \mu \mathrm{M}$ 
of fresh Chiron daily. Samples were analyzed via flow cytometry on day 4 and 7 for expression of CD4, CD25, CD3, $\beta$-catenin, Foxp3 and RORyt. For the TCR/wnt3a stimulation $4 \times 10^{6}$ cells were seeded in 6well plates. They were stimulated with human T-activator CD3/CD28 Dynabeads (Thermo Fisher, 11161D) at a 1:1 bead-to-cell ratio and rh-wnt3a (R\&D Systems, 5036-WN-010) was added at $100 \mathrm{ng} / \mathrm{ml}$ dilution. Cells were supplemented with $50 \mathrm{ng} / \mathrm{ml}$ rh-Wnt3a daily. On day 4, the T-activator beads were removed, while wnt3a treatment continued until day 6 . On day 6 , cells were split, and one half was restimulated with Cell Stimulation Cocktail for 3 hours. All samples were analyzed by flow cytometry using the same panel as for the patient sample analysis and spectral flow cytomerty.

Mice. Foxp3 ${ }^{\text {YFP-Cre }}$ Ctnnb1 $1^{\mathrm{fl}(\mathrm{ex} 3)} 49$, Foxp3 ${ }^{\mathrm{YFP}-\mathrm{Cre} 50}{ }^{50}, \mathrm{APC}^{\triangle 468} 43, \mathrm{CD} 45.1$ and WT mice were used in all experiments described. All mice were maintained on the C57BL/ 6 background. Mice were housed under specific pathogen-free (SPF) conditions in the animal facilities at the University of Chicago in accordance with protocol \#71880, approved by the University of Chicago Institutional Animal Care and Use Committee.

Mouse model for IBD-associated colon cancer. 3-4 months old APC ${ }^{\triangle 468}$ (male and female) and agedmatched WT mice were treated with 3 rounds of $2 \%$ dextran sulfate sodium salt (colitis grade, 160110, $\mathrm{MP}$ ) in the drinking water ad libitum over 7 days with a 2-week recovery period on normal drinking water between each round (see experimental treatment scheme Fig. 3a). Weight loss was monitored every other day.

Histological assessment of inflamed tissues. Peripheral lymphoid organs and vital organs were resected from mice and directly ex vivo formalin fixed (HT5014, Sigma-Aldrich). Colons were harvested from mice, flushed free of feces with ice-cold HBBS, formalin fixed, and Swiss rolled. All fixed organs were paraffin embedded and $4 \mathrm{~mm}$ sections were used for H\&E staining. Embedding, sectioning and H\&E staining service was provided by the University of Chicago Human Tissue Resource Center (HTRC).

\section{Isolation of lymphocytes from thymi, peripheral lymphoid organs, and the intestinal mucosa of mice.}

Thymi, peripheral lymph nodes, mesenteric lymph nodes, and spleens were resected. Single cell suspensions were generated by mashing the organs through 40-100 $\mu \mathrm{m}$ cells strainers while flushing the strainers with ice-cold $1 \times$ PBS. Erythrocytes in the spleen single cell suspensions were lysed by resuspending the cell pellets in $2 \mathrm{ml} \mathrm{ACK}$ buffer $\left(150 \mathrm{mM} \mathrm{NH}_{4} \mathrm{Cl}, 10 \mathrm{mM} \mathrm{KHCO}_{3}, 0,1 \mathrm{mM}\right.$ EDTA, $\mathrm{pH}=7,2-$ 74), each, for $1 \mathrm{~min}$ on ice. The reaction was stopped by adding $15 \mathrm{ml}$ of PBS $+2 \%$ FCS, cells were spun down immediately after and washed in $10 \mathrm{ml}$ ice cold PBS. Dead cells were removed by filtering the lysed cells suspensions through $40 \mu \mathrm{m}$ cell strainers. Intestinal lamina propria lymphocytes were isolated according to the protocol published by Atarashi and colleagues ${ }^{69}$.

$\mathrm{T}_{\text {reg }}$ Proliferation Inhibition Assay. Untouched $\mathrm{CD} 4^{+} \mathrm{CD} 25^{-} \mathrm{T}$ cells were isolated from peripheral lymph nodes and spleens of 10-week old CD45.1 mice using the CD $4^{+} \mathrm{T}$ cell isolation kit, mouse (130-104-454, Miltenyi). The protocol was modified by adding $1 \mu$ l biotin rat anti-mouse $\operatorname{CD} 25(553070, B D)$ antibody per $100 \mu$ l of the Labeling Cocktails to remove $T_{\text {regs. }}$. Purified CD $4^{+}$CD $25^{-}$CD $45.1^{+} T$ cells were CFSE 
labeled using Cell Trace CFSE (C34554, Invitrogen/Fisher) and stimulated using Dynabeads Mouse TActivation CD3/CD28 (11456-D, Gibco/Life Technologies/Fisher) in a 1:1 ratio. 50,000 CFSE-labeled T cells and 50,000 beads were seeded in 96-well round-bottom plates in $200 \mu$ l culture media (DMEM (D6429, Sigma) supplemented with $0.4 \%$ Gentamycin (G1397, Sigma), 1\% HEPES, and 10\%FCS per well. After 48h, $C D 4^{+} \mathrm{CD} 25^{+} \mathrm{T}_{\text {reg }}$ s from Foxp3 ${ }^{\mathrm{YFP}-\mathrm{Cre}} \mathrm{Ctnnb} 1^{\mathrm{fl}(\mathrm{ex} 3)}$ and Foxp3 ${ }^{\mathrm{YFP}-\mathrm{Cre}}$ mice were added, respectively. $T_{\text {reg }}$ s were purified from peripheral lymph nodes and spleens using the CD4+CD25+ Regulatory T cell Isolation Kit II (130-091-041, Miltenyi). The assay was performed in triplicates with $\mathrm{T}_{\text {reg }}$ to $T$ effector ratios of 1:1,1:2, and 1:4. Samples were incubated for another $48 \mathrm{~h}$ and then collected for flow cytometric read out. Cells were stained for viability and then surface stained with CD45.1 (clone A20, BD), CD45.2 (clone 104, BD), CD25 (clone PC61, BD) and CD4 (clone GK1.5, BD) antibodies. Stained samples were acquired using a 4 laser/15 detector LSR-Fortessa instrument (BD).

Flow cytometry and FACS of murine lymphocytes. Cells isolated from peripheral lymphoid organs, thymi, and intestinal mucosa were either first re-stimulated with Cell Stimulation Cocktail (plus protein inhibitors, $500 \mathrm{x}$, eBioscience, 00-4975-93) for three hours in X-Vivo-20 (LONZA, 04-448-Q) or used directly for flow cytometric staining. Cells were stained for viability using the LIVE/DEAD Aqua fluorescent reactive dye (Molecular Probes - life technologies, L34963), followed by surface staining with varying combinations the following antibodies in FACS buffer (1x PBS + 2\% v/v FCS): CD3 (clone 145-2C11, BD), CD4 (clone GK1.5, BD), CD8 (clone 53-6.7, BD), CD25 (clone PC61, BD), CD44 (clone IM7, BD), CD62L (clone MEL-14, BD), CD69 (clone H1.2F3, BD), CD103 (clone M290, BD), CD196/CCR6 (clone 29-2L17, BioLegend), CD199/CCR9 (clone CW-1.2, BioLegend), CD304/Neuropilin-1 (clone 3E12, BioLegend), CTLA-4 (UC104B9, BioLegend), GITR (DTA-1, BD). Following surface staining, cells were fixed and permeabilized using Foxp3/Transcription Factor Fixation/Permeabilization Concentrate and Diluent (Affymetrix/eBioscience/Thermo Fisher Scientific, 00-5521). Intracellular flow cytometric staining was performed with anti- $\beta$-catenin (clone 14/Beta-Catenin, BD), RORyt (clone Q31-378, BD), Foxp3 (clone FJK16s, eBiosience/Fisher), Helios (clone 22F6, BioLegend), anti-GFP (clone FM264C, BioLegend), IFNy (clone XMG1.2, BD), IL-17A (clone TC11-18H10, BD), TNFa (MP6-XT22, BioLegend), Ki67 (B56, BD), and respective manufacturer isotype control antibodies. Stained samples were acquired using a 5 laser/18 detector LSR-Fortessa X20 (BD) instrument, an 5-Laser Aurora (Cytek ${ }^{\mathrm{TM}}$ ) instrument, or sorted using 4 laser/15 detector Ariall/Illu (BD) FACS sorters. For the spectral flow stainings, Brilliant Stain Buffer Plus (BD) was added to the staining mixes according to manufacturer's insctructions. Flow data was analyzed using FlowJo Version 10 software (FlowJo LLC, BD).

Chromatin immunoprecipitation and sequencing (ChIP-seq). $1 \times 10^{7} \mathrm{CD}^{2} 5^{+} \mathrm{YFP}^{+} \mathrm{T}_{\text {reg }}$ s were FACS sorted from LN and SPL isolated CD4 ${ }^{+}$T cells (CD4 ${ }^{+}$T Cell Isolation Kit, mouse, 130-104-454, Miltenyi) of 10-12week-old Foxp3 ${ }^{\text {YFP-Cre }}$ WT mice. Chromatin immunoprecipitation and library generation performed as previously described ${ }^{14,70}$. Libraries were sequenced on a HiSeq 4000 sequencer at the University of Chicago Genomics Facility. 
Isolation and preparation of samples for RNA-seq. 1.5-4.0x $10^{5} \mathrm{CD} 25^{+} \mathrm{YFP}^{+} \mathrm{T}_{\text {reg }}$ s were FACS sorted from MACS-pre-purified CD4 ${ }^{+} \mathrm{T}$ cells (CD4 ${ }^{+} \mathrm{T}$ Cell Isolation Kit, mouse, 130-104-454, Miltenyi) isolated from LNs of 21 day old Foxp3 ${ }^{\text {YFP-Cre }} \mathrm{Ctnnb}^{\mathrm{fl}(\mathrm{ex} 3)}$ and Foxp3 ${ }^{\text {YFP-Cre } W T}$ mice. Total RNA was isolated using the PicoPure ${ }^{\text {TM }}$ RNA Isolation Kit (KIT0202, Arcturus) following the manufacturer's instructions. Libraries were generated and sequenced by the University of Chicago Genomics Facility.

\section{Assay for transposase accessible chromatin and sequencing (ATAC-seq). For ATAC-seq, 5-10x10 4} $\mathrm{YFP}^{+} \mathrm{CD} 25^{+} \mathrm{T}_{\text {reg }}$ s were FACS sorted from MACS-pre-purified CD 4 ${ }^{+} \mathrm{T}$ cells (CD4+ T Cell Isolation Kit, mouse, 130-104-454, Miltenyi) isolated from LNs of 21 day old Foxp3 ${ }^{\text {YFP-Cre }}{ }^{-C t n n b} 1^{\mathrm{fl}(\mathrm{ex} 3)}$ and Foxp3 ${ }^{\mathrm{YFP}-\mathrm{Cre}}$ WT mice. Cells were centrifuged at $500 \mathrm{~g}$ at $4^{\circ} \mathrm{C}$ for $5 \mathrm{~min}$, washed with $1 \times \mathrm{PBS}$, and centrifuged again. Cells were resuspended in lysis buffer $(10 \mathrm{mM}$ Tris- $\mathrm{HCl}, \mathrm{pH} 7.4,10 \mathrm{mM} \mathrm{NaCl}, 3 \mathrm{mM} \mathrm{MgCl}$, and $0.1 \%$ IGEPAL CA-630) and immediately centrifuged at $500 \mathrm{~g}$ at $4{ }^{\circ} \mathrm{C}$ for $10 \mathrm{~min}$. Pellets were resuspended in transposition reaction buffer (25 $\mu \mathrm{l} 2 \times$ Tagment Buffer (FC-121-1030, Illumina), $2.5 \mu \mathrm{l}$ Tagment DNA Enzyme, and $22.5 \mu \mathrm{l}$ nuclease-free $\mathrm{H}_{2} \mathrm{O}$ ) for 30 min at $37^{\circ} \mathrm{C}$. DNA was purified with a Qiagen MinElute Kit and amplified with Nextera PCR primers (Illumina Nextera Index Kit) and NEBNext PCR Master Mix (M0541, New England BioLabs). Amplified DNA was purified with a Qiagen PCR cleanup kit. Libraries were sequenced on a HiSeq 4000 sequencer at the University of Chicago Genomics Facility.

Genome mapping and data analysis. Sequenced ChIP and ATAC datasets were mapped with the Galaxy (https://usegalaxy.org/) suite of tools. Data were groomed and aligned to the mouse mm10 genome with Bowtie, allowing up to one mismatch and retaining only uniquely mapped reads, and unmapped reads were filtered. For transcription factors (TCF-1, Foxp3) peak calling was performed with MACS via $\mathrm{HOMER}^{71}$. Transcription factor peak calling was performed relative to input controls with the requirement that peaks be at a minimum 5-fold enriched over input and meet a p-value cutoff of $1 \times 10^{-5}$. Openchromatin (ATAC-seq) peaks were called with Macs $2^{72}$ with -nomodel set and no background provided. Differential accessibility testing of ATACseq data was performed using the edgeR Bioconductor package $^{73}$. Sequenced RNA datasets were aligned to the mouse $\mathrm{mm} 10$ genome like the ChIP-seq datasets. Differential gene expression analysis was performed with Cuffdiff $2^{74}$. Genes with transcript abundance differences below $\mathrm{q}<0.05$ were considered to be significantly differentially expressed. Heat maps of normalized reads for gene subsets were generated with using Genepattern ${ }^{75}$. Motif analysis was performed with the HOMER motif-discovery algorithm, and transcription-factor overlap analysis was conducted with the HOMER mergePeaks command, considering only peaks that directly overlapped. Peaks were annotated to the mm10 genome with annotatePeaks.pl in HOMER. Histograms for transcription factors and histone modifications were generated with ngs.plot software ${ }^{76}$. K-means clustering of ChIP-seq datasets and heat maps were also generated with ngs. plot. Transcription factor binding was visualized with the Integrated Genome Browser software ${ }^{77}$. Pathway enrichment analysis for genes identified by ChIP-seq and RNA-seq analysis was performed via Metascape (http://metascape.org) ${ }^{78}$. 
Statistical analysis. Results from biologically distinct experiments were combined and analyzed with the indicated statistical tests in Prism 7 (GraphPad). The statistical significance of RNA-seq data was determined with Cuffdiff and for ATACseq data with edgeR. ChIP-seq (factor enrichment) and ATAC-seq (chromatin accessibility) p-value cutoffs were determined with MACS2. Gene pathway enrichment pvalues were determined with Metascape. Data are presented as mean \pm SEM.

\section{References}

1. Kuswanto, W. et al. Poor Repair of Skeletal Muscle in Aging Mice Reflects a Defect in Local, Interleukin33-Dependent Accumulation of Regulatory T Cells. Immunity 44, 355-367 (2016). 2. Ito, M. et al. Brain regulatory T cells suppress astrogliosis and potentiate neurological recovery. Nature 565, 246-250 (2019). 3. Zhu, J., Yamane, H. \& Paul, W.E. Differentiation of effector CD4 T cell populations ( $\left.{ }^{*}\right)$. Annual review of immunology 28, 445-489 (2010). 4. Levine, A.G. et al. Stability and function of regulatory T cells expressing the transcription factor T-bet. Nature 546, 421-425 (2017). 5. Yu, F., Sharma, S., Edwards, J., Feigenbaum, L. \& Zhu, J. Dynamic expression of transcription factors T-bet and GATA-3 by regulatory T cells maintains immunotolerance. Nature immunology 16, 197-206 (2015). 6. Ohnmacht, C. et al. MUCOSAL IMMUNOLOGY. The microbiota regulates type 2 immunity through RORgammat(+) T cells. Science 349, 989-993 (2015). 7. Sefik, E. et al. MUCOSAL IMMUNOLOGY. Individual intestinal symbionts induce a distinct population of RORgamma(+) regulatory T cells. Science 349, 993-997 (2015). 8. Yang, B.H. et al. Foxp3(+) T cells expressing RORgammat represent a stable regulatory T-cell effector lineage with enhanced suppressive capacity during intestinal inflammation. Mucosal Immunol 9, 444-457 (2016). 9. Samstein, R.M. et al. Foxp3 exploits a pre-existent enhancer landscape for regulatory T cell lineage specification. Cell 151, 153-166 (2012). 10. Fu, W. et al. A multiply redundant genetic switch 'locks in' the transcriptional signature of regulatory T cells. Nature immunology 13, 972-980 (2012). 11. Delacher, M. et al. Genome-wide DNA-methylation landscape defines specialization of regulatory $T$ cells in tissues. Nature immunology 18, 1160-1172 (2017). 12. Kwon, H.K., Chen, H.M., Mathis, D. \& Benoist, C. Different molecular complexes that mediate transcriptional induction and repression by FoxP3. Nature immunology 18, 1238-1248 (2017). 13. Xing, S. et al. Tcf1 and Lef1 are required for the immunosuppressive function of regulatory $T$ cells. The Journal of experimental medicine 216, 847-866 (2019). 14. Emmanuel, A.O. et al. TCF-1 and HEB cooperate to establish the epigenetic and transcription profiles of CD4(+)CD8(+) thymocytes. Nature immunology 19, 1366-1378 (2018). 15. Choi, Y.S. et al. LEF1 and TCF-1 orchestrate $\mathrm{T}(\mathrm{FH})$ differentiation by regulating differentiation circuits upstream of the transcriptional repressor Bcl6. Nature immunology 16, 980-990 (2015). 16. Zhou, X. et al. Differentiation and persistence of memory CD8(+) T cells depend on T cell factor 1. Immunity 33, 229-240 (2010). 17. Scott, A.C. et al. TOX is a critical regulator of tumour-specific T cell differentiation. Nature 571, 270-274 (2019). 18. MacDonald, B.T., Tamai, K. \& He, X. Wnt/beta-catenin signaling: components, mechanisms, and diseases. Dev Cell 17, 9-26 (2009). 19. Lovatt, M. \& Bijlmakers, M.J. Stabilisation of beta-catenin downstream of T cell receptor signalling. PloS one 5 (2010). 20. van Loosdregt, J. et al. Canonical Wnt signaling negatively modulates regulatory $T$ cell function. Immunity 39, 298-310 (2013). 21. Sumida, T. et al. Activated beta-catenin in Foxp3(+) regulatory T cells links inflammatory environments to 
autoimmunity. Nature immunology 19, 1391-1402 (2018). 22. Mittal, D., Gubin, M.M., Schreiber, R.D. \& Smyth, M.J. New insights into cancer immunoediting and its three component phases-elimination, equilibrium and escape. Current opinion in immunology 27, 16-25 (2014). 23. Wing, J.B., Tanaka, A. \& Sakaguchi, S. Human FOXP3(+) Regulatory T Cell Heterogeneity and Function in Autoimmunity and Cancer. Immunity 50, 302-316 (2019). 24. Salama, P. et al. Tumor-infiltrating FOXP3+ T regulatory cells show strong prognostic significance in colorectal cancer. Journal of clinical oncology : official journal of the American Society of Clinical Oncology 27, 186-192 (2009). 25. deLeeuw, R.J., Kost, S.E., Kakal, J.A. \& Nelson, B.H. The prognostic value of FoxP3+ tumor-infiltrating lymphocytes in cancer: a critical review of the literature. Clinical cancer research : an official journal of the American Association for Cancer Research 18, 3022-3029 (2012). 26. Sinicrope, F.A. et al. Intraepithelial effector (CD3+)/regulatory (FoxP3+) T-cell ratio predicts a clinical outcome of human colon carcinoma. Gastroenterology 137, 12701279 (2009). 27. Saito, T. et al. Two FOXP3(+)CD4(+) T cell subpopulations distinctly control the prognosis of colorectal cancers. Nature medicine 22, 679-684 (2016). 28. Feagins, L.A., Souza, R.F. \& Spechler, S.J. Carcinogenesis in IBD: potential targets for the prevention of colorectal cancer. Nat Rev Gastroenterol Hepatol 6, 297-305 (2009). 29. Terzic, J., Grivennikov, S., Karin, E. \& Karin, M. Inflammation and colon cancer. Gastroenterology 138, 2101-2114 e2105 (2010). 30. Blatner, N.R. et al. Expression of RORgammat marks a pathogenic regulatory $T$ cell subset in human colon cancer. Science translational medicine 4, 164ra159 (2012). 31. Keerthivasan, S. et al. beta-Catenin promotes colitis and colon cancer through imprinting of proinflammatory properties in T cells. Science translational medicine 6, 225ra228 (2014). 32. Miyara, M. et al. Functional delineation and differentiation dynamics of human CD4+ T cells expressing the FoxP3 transcription factor. Immunity 30, 899-911 (2009). 33. Kanehisa, M. \& Goto, S. KEGG: kyoto encyclopedia of genes and genomes. Nucleic acids research 28, 27-30 (2000). 34. Kanehisa, M., Furumichi, M., Tanabe, M., Sato, Y. \& Morishima, K. KEGG: new perspectives on genomes, pathways, diseases and drugs. Nucleic acids research 45, D353-D361 (2017). 35. Tosolini, M. et al. Clinical impact of different classes of infiltrating T cytotoxic and helper cells (Th1, th2, treg, th17) in patients with colorectal cancer. Cancer research 71, 1263-1271 (2011). 36. Beurel, E. \& Jope, R.S. Differential regulation of STAT family members by glycogen synthase kinase-3. The Journal of biological chemistry 283, 2193421944 (2008). 37. Beurel, E., Michalek, S.M. \& Jope, R.S. Innate and adaptive immune responses regulated by glycogen synthase kinase-3 (GSK3). Trends Immunol 31, 24-31 (2010). 38. Beurel, E., Yeh, W.I., Michalek, S.M., Harrington, L.E. \& Jope, R.S. Glycogen synthase kinase-3 is an early determinant in the differentiation of pathogenic Th17 cells. Journal of immunology 186, 1391-1398 (2011). 39. Wei, L., Laurence, A., Elias, K.M. \& O'Shea, J.J. IL-21 is produced by Th17 cells and drives IL-17 production in a STAT3-dependent manner. The Journal of biological chemistry 282, 34605-34610 (2007). 40. Beurel, E. et al. Regulation of Th1 cells and experimental autoimmune encephalomyelitis by glycogen synthase kinase-3. Journal of immunology 190, 5000-5011 (2013). 41. O'Shea, J.J. \& Paul, W.E. Mechanisms underlying lineage commitment and plasticity of helper CD4+ T cells. Science 327, 1098-1102 (2010). 42. Kovalovsky, D. et al. Beta-catenin/Tcf determines the outcome of thymic selection in response to alphabetaTCR signaling. Journal of immunology 183, 3873-3884 (2009). 43. Gounari, F. et al. Loss of adenomatous polyposis coli gene function disrupts thymic development. Nature immunology 6, 800-809 (2005). 44. Moser, A.R. et al. ApcMin: a mouse model for intestinal and mammary tumorigenesis. Eur J 
Cancer 31A, 1061-1064 (1995). 45. You, S. et al. Developmental abnormalities in multiple proliferative tissues of Apc(Min/+) mice. Int J Exp Pathol 87, 227-236 (2006). 46. Chassaing, B., Aitken, J.D., Malleshappa, M. \& Vijay-Kumar, M. Dextran sulfate sodium (DSS)-induced colitis in mice. Curr Protoc Immunol 104, Unit 1525 (2014). 47. Cooper, H.S. et al. The role of mutant Apc in the development of dysplasia and cancer in the mouse model of dextran sulfate sodium-induced colitis. Gastroenterology 121, 1407-1416 (2001). 48. Tanaka, T. et al. Dextran sodium sulfate strongly promotes colorectal carcinogenesis in Apc(Min/+) mice: inflammatory stimuli by dextran sodium sulfate results in development of multiple colonic neoplasms. International journal of cancer. Journal international du cancer 118, 25-34 (2006). 49. Harada, N. et al. Intestinal polyposis in mice with a dominant stable mutation of the beta-catenin gene. Embo J 18, 5931-5942 (1999). 50. Rubtsov, Y.P. et al. Regulatory T cellderived interleukin-10 limits inflammation at environmental interfaces. Immunity 28, 546-558 (2008). 51. Godfrey, V.L., Wilkinson, J.E. \& Russell, L.B. X-linked lymphoreticular disease in the scurfy (sf) mutant mouse. Am J Pathol 138, 1379-1387 (1991). 52. Brunkow, M.E. et al. Disruption of a new forkhead/winged-helix protein, scurfin, results in the fatal lymphoproliferative disorder of the scurfy mouse. Nature genetics 27, 68-73 (2001). 53. Wing, K. et al. CTLA-4 control over Foxp3+ regulatory T cell function. Science 322, 271-275 (2008). 54. Franckaert, D. et al. Promiscuous Foxp3-cre activity reveals a differential requirement for CD28 in Foxp3(+) and Foxp3(-) T cells. Immunology and cell biology 93, 417423 (2015). 55. Kitagawa, Y. et al. Guidance of regulatory T cell development by Satb1-dependent superenhancer establishment. Nature immunology 18, 173-183 (2017). 56. Subramanian, A. et al. Gene set enrichment analysis: a knowledge-based approach for interpreting genome-wide expression profiles. Proceedings of the National Academy of Sciences of the United States of America 102, 15545-15550 (2005). 57. Zitvogel, L., Ayyoub, M., Routy, B. \& Kroemer, G. Microbiome and Anticancer Immunosurveillance. Cell 165, 276-287 (2016). 58. Voloshanenko, O. et al. Wnt secretion is required to maintain high levels of Wnt activity in colon cancer cells. Nat Commun 4, 2610 (2013). 59. Moparthi, L. \& Koch, S. Wnt signaling in intestinal inflammation. Differentiation (2019). 60. Pham, T.H., Okada, T., Matloubian, M., Lo, C.G. \& Cyster, J.G. S1P1 receptor signaling overrides retention mediated by G alpha icoupled receptors to promote T cell egress. Immunity 28, 122-133 (2008). 61. Turner, J.R. Intestinal mucosal barrier function in health and disease. Nature reviews. Immunology 9, 799-809 (2009). 62. Konig, J. et al. Human Intestinal Barrier Function in Health and Disease. Clin Transl Gastroenterol 7, e196 (2016). 63. Schmidt, H.H. et al. HLA Class II tetramers reveal tissue-specific regulatory T cells that suppress T-cell responses in breast carcinoma patients. Oncoimmunology 2, e24962 (2013). 64. Rathinasamy, A. et al. Tumor specific regulatory $T$ cells in the bone marrow of breast cancer patients selectively upregulate the emigration receptor S1P1. Cancer immunology, immunotherapy : Cll 66, 593603 (2017). 65. Hoerl, A.E. \& Kennard, R.W. Ridge Regression: Biased Estimation for Nonorthogonal Problems. Technometrics 12, 55-67 (1970). 66. Therneau, T.M. A Package for Survival Analysis in S. 2.38 ed. CRAN.R-project.org; 2015. 67. Friedman, J.H., Hastie, T. \& Tibshirani, R. Regularization Paths for Generalized Linear Models via Coordinate Descent. Journal of statistical software 33, 22 (2010). 68. Kassambara, A., Kosinski, M., Biecek, P. \& Fabian, S. Package 'survminer'. 2017. 69. Atarashi, K. et al. Th17 Cell Induction by Adhesion of Microbes to Intestinal Epithelial Cells. Cell 163, 367-380 (2015). 70. Dose, M. et al. beta-Catenin induces T-cell transformation by promoting genomic instability. Proceedings 
of the National Academy of Sciences of the United States of America 111, 391-396 (2014). 71. Heinz, S. et al. Simple combinations of lineage-determining transcription factors prime cis-regulatory elements required for macrophage and B cell identities. Molecular cell 38, 576-589 (2010). 72. Zhang, Y. et al. Model-based analysis of ChIP-Seq (MACS). Genome Biol 9, R137 (2008). 73. Robinson, M.D., McCarthy, D.J. \& Smyth, G.K. edgeR: a Bioconductor package for differential expression analysis of digital gene expression data. Bioinformatics 26, 139-140 (2010). 74. Trapnell, C. et al. Differential gene and transcript expression analysis of RNA-seq experiments with TopHat and Cufflinks. Nat Protoc 7, 562-578 (2012). 75. Reich, M. et al. GenePattern 2.0. Nature genetics 38, 500-501 (2006). 76. Shen, L., Shao, N., Liu, X. \& Nestler, E. ngs.plot: Quick mining and visualization of next-generation sequencing data by integrating genomic databases. BMC Genomics 15, 284 (2014). 77. Freese, N.H., Norris, D.C. \& Loraine, A.E. Integrated genome browser: visual analytics platform for genomics. Bioinformatics 32, 2089-2095 (2016). 78. Tripathi, S. et al. Meta- and Orthogonal Integration of Influenza "OMICs" Data Defines a Role for UBR4 in Virus Budding. Cell Host Microbe 18, 723-735 (2015).

\section{Figures}




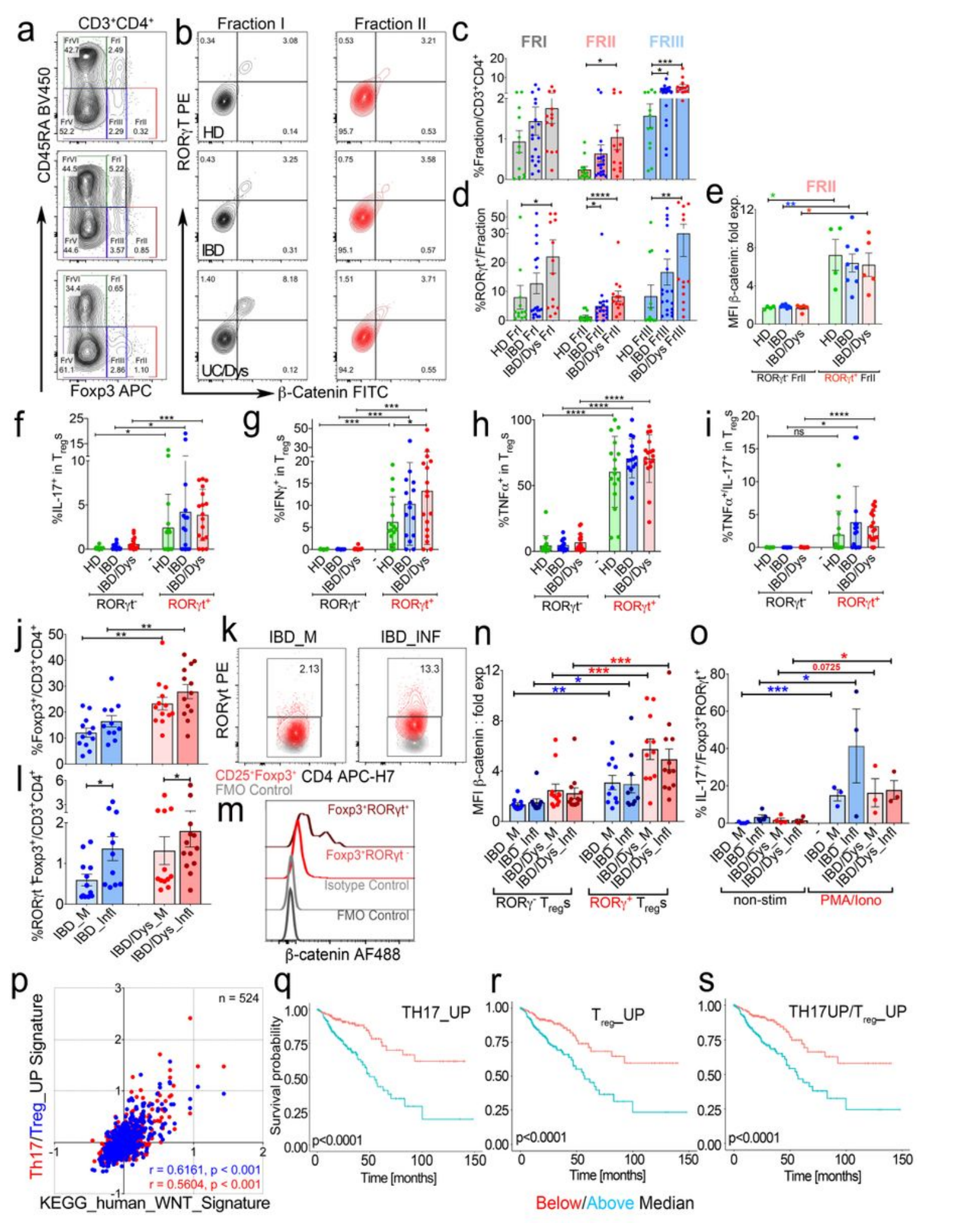

\section{Figure 1}

$\beta$-cateninhigh RORyt+ Tregs producing IL-17, IFNy and TNFa expand in the PB and colonic mucosa of IBD patients during malignant disease progression. a. Live CD3+CD4+ cells were divided into 5 fractions according to their CD45RA versus Foxp3 expression. b. RORyt versus B-catenin expression assessed in Treg fractions Fr.I and Fr.II. Representative samples are shown for HD (HD8), and for IBD (IMB11) and IBD/Dys (IMB6) patients. c. Cumulative analysis of Treg fraction (Fr.I, Fr.II, Fr.III) frequencies in PB of HDs, 
IBD, and IBD/Dys patients and d. relative frequencies of RORyt+ cells within individual Treg fractions (Kruskal-Wallis test). Error analysis: SEM. e. Cumulative $\beta$-catenin expression of PB RORYt+/RORyt- Fr.II Tregs, depicted as MFI normalized to Isotype/FMO controls for individual patients (paired t-test). f. IL-17 g. IFNy h. TNFa and i. IL-17+TNFa production after stimulation with PMA/lonomycin by RORyt- and RORyt+ CD4+CD25+Foxp3+CD127- PB Tregs assessed by intracellular cytokine staining (paired t-test). f-i. $n(H D)=16, n(I B D)=15, n(I B D / D y s)=17$. j. Cumulative frequencies of $C D 3+C D 4+F o x p 3+$ cells in the inflamed (Infl) and less inflamed (margin, M) mucosa of IBD and IBD/Dys patients. k. Flow plots and, I. cumulative assessment of Foxp3+RORyt+Treg frequencies. m. Flow-cytometric histogram and, $n$. cumulative $\beta$-catenin expression of RORYt+/RORyt- Tregs, depicted as normalized MFI (paired t-test). 0 . IL-17 production before (non-stim) and after stimulation (PMA/lono) of mucosal Treg populations assessed by intracellular cytokine staining (unpaired t-test). $k-0 . n\left(I B D \_M\right) \leq 12, n\left(I B D \_I n f l\right) \leq 11$, $n\left(I B D / D y s \_M\right) \leq 13, n\left(I B D / D y s \_I n f l\right) \leq 13$. Analysis of the TCGA CRC cancer cohort: $p$. Spearman correlation of average $z$-scores for the KEGG_human_WNT versus signature Th17_UP (red, $r=0.5604$, $p<0.001)$ and Treg_UP (blue, $r=0.6161, p<0.001)$ signature in CRC patients $(n=524)$. q. Kaplan-Meier plots based on dichotomizing patient-levels of, p. TH17_UP and Treg_UP, and, q-s. the combined TH17_UP/Treg_UP signature-based scores on the median ( $p<0.001$, log-rank test). 
a

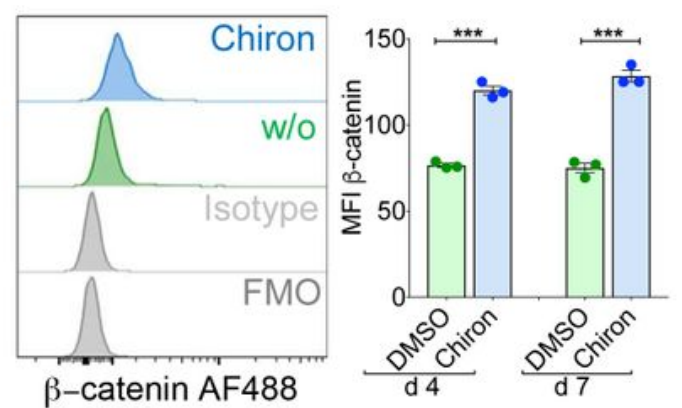

Day 0

e

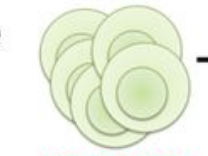

HD PBMCs b wnt3a CD3/CD28 beads

TGF $\beta / L-4 / L-2$
C

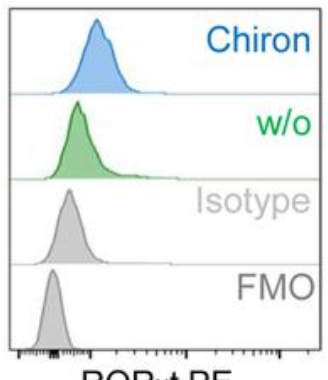

d

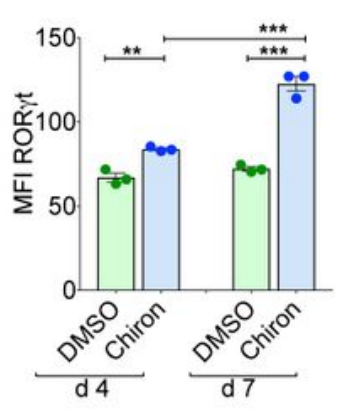

Day 4

RORyt PE

Day 6
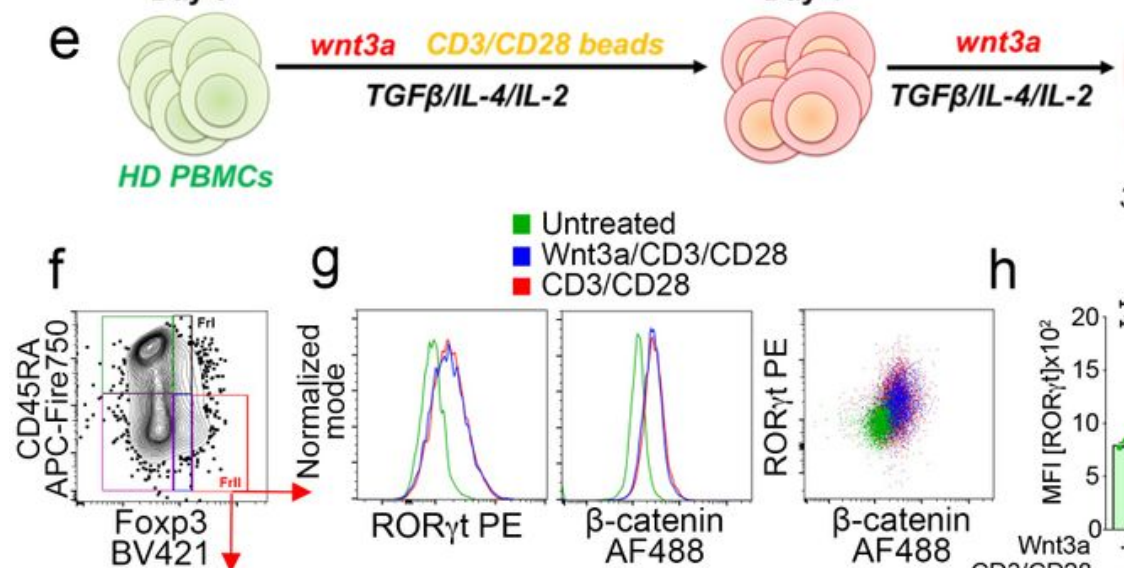

/CD28

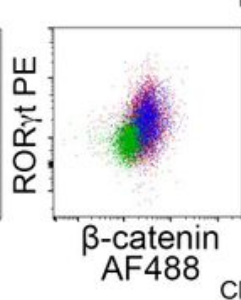

h

3 h PMAVlono

Analysis

h
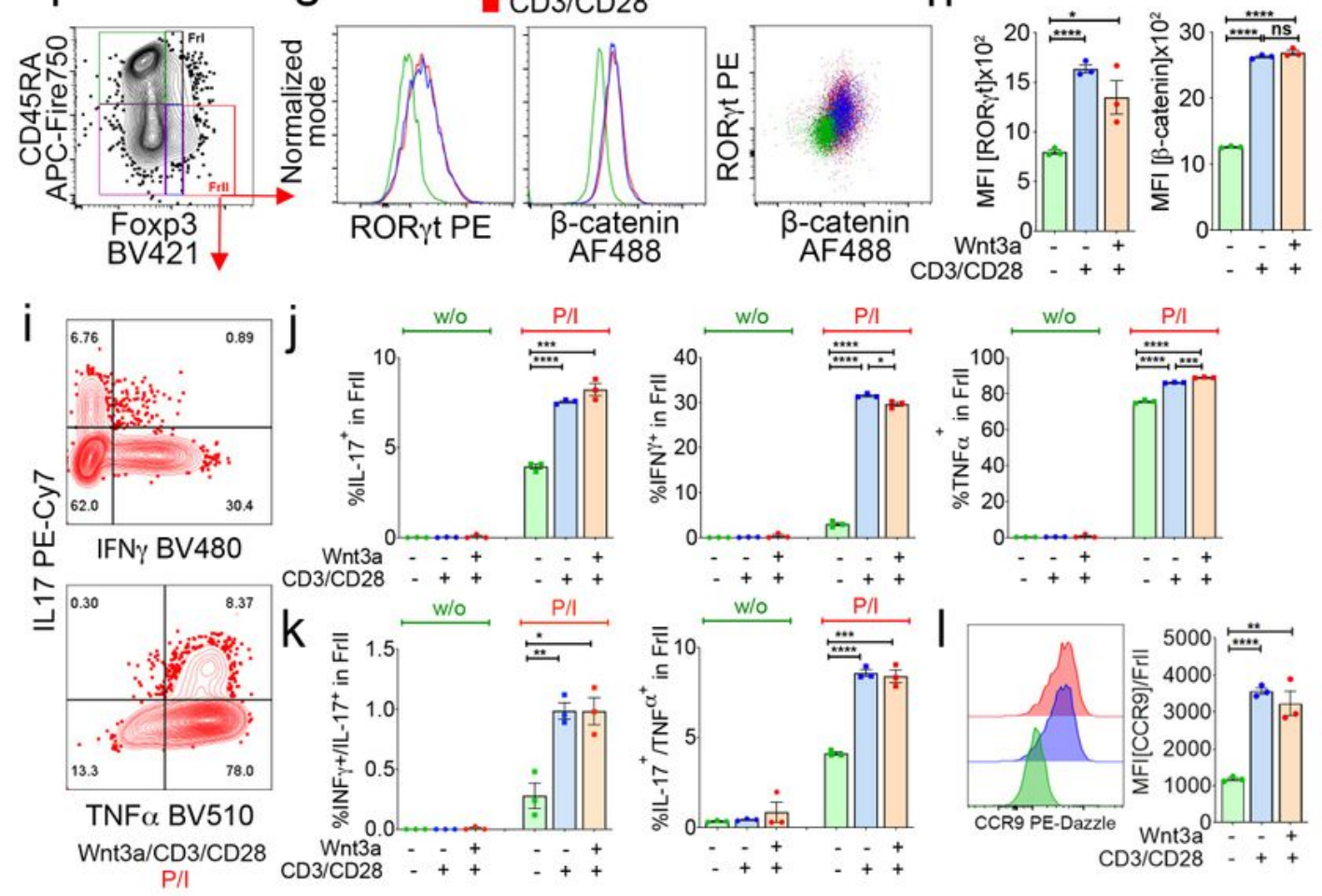

\section{Figure 2}

Ex vivo stabilization of $\beta$-catenin in human Tregs is sufficient to induce the pro-inflammatory phenotype. a-d, Ex vivo treatment of HD PBMCs with GSK-3 $\beta$ inhibitor Chiron for 4 to 7d. Flow cytometric histograms and cumulative MFI analysis of $\beta$-catenin, a-b, and RORyt, c, d, expression in CD25+Foxp3+ Tregs cells in these cultures. e-l, Ex vivo Wnt signaling pathway induction in healthy donor PBMCs. e, Scheme of the experimental set-up of ex vivo Wnt signaling induction in HD PBMCs. f, Representative gating scheme to 
identify Tregs according to Sakaguchi and colleagues $\mathrm{g}$, Representative flow cytometric histograms and dot plot of $\beta$-catenin and RORyt expression in Fr.II Tregs on day 6 of culture. h, Cumulative MFI analysis of $\beta$-catenin and RORyt expression in Tregs. i, Representative flow cytomeric profiles of IL-17 versus INFY or TNFa production in Fr.II Tregs. j, Cumulative analysis of IL-17+, INFY+, and TNFa+ Fr.II Tregs and k, dual production of IL-17/INFY and IL-17/TNFa before an after stimulation with PMA/lonomycin (P/I). I, Represenative flow cytometric histograms and cumulative MFI analysis of CCR9 expression. Each experiment was performed 3 times (with differing HDs' PBMCs); statistical testing: unpaired t-test; error analysis: SEM. 


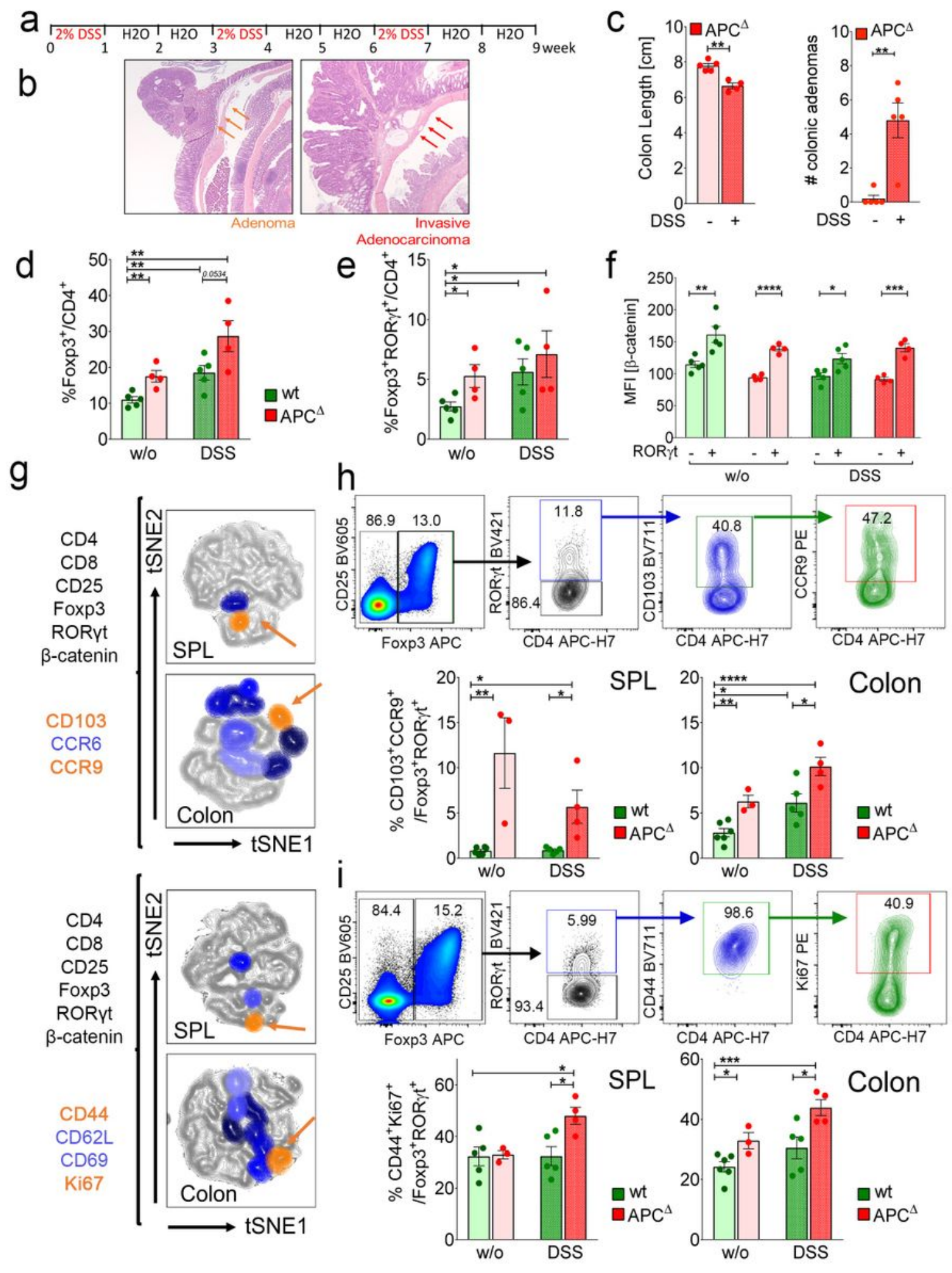

Figure 3

Highly activated and gut homing RORyt+ Treg sub-populations peripherally expanded during disease progression in a murine IBD/CRC model. a, Experimental treatment scheme for WT and APC $\triangle$ mice. b-c, Histological assessment of DSS-treated and untreated APC $\triangle$ mice. b, Representative H\&E staining of an adenoma and an invasive adenocarcinoma. c, Colon length and adenoma numbers in the colon of DSS treated and untreated APC $\triangle$ mice. d, Frequencies of colon-resident Foxp3+ Tregs and, e, RORyt+ Tregs 
within CD4+CD8- T cells for the 4 different treatment groups. $f, \beta$-catenin expression of RORyt+ versus RORyt-Treg populations. g, Identification and characterization of RORyt+ Treg subpopulations that increase in frequency during disease progression via tSNE-projection of multi-color flow-cytometric data; two independent flow-cytometric panels characterize migratory behavior and activation status. Orange arrows indicate shared populations between SPL and colon. Gating for andquantification of shared RORyt+ Treg populations in different anatomic locations and treatment groups for the $h$, migratory and $i$, activation flow-cytometric panels. $S P L=$ spleen; $n(W T)=6, n(W T+D S S)=5, n(A P C \Delta)=4, n(A P C \Delta+D S S)$ $=4,1$ out of a series of 3 experiments, statistical testing: unpaired t-test, error analysis: SEM 

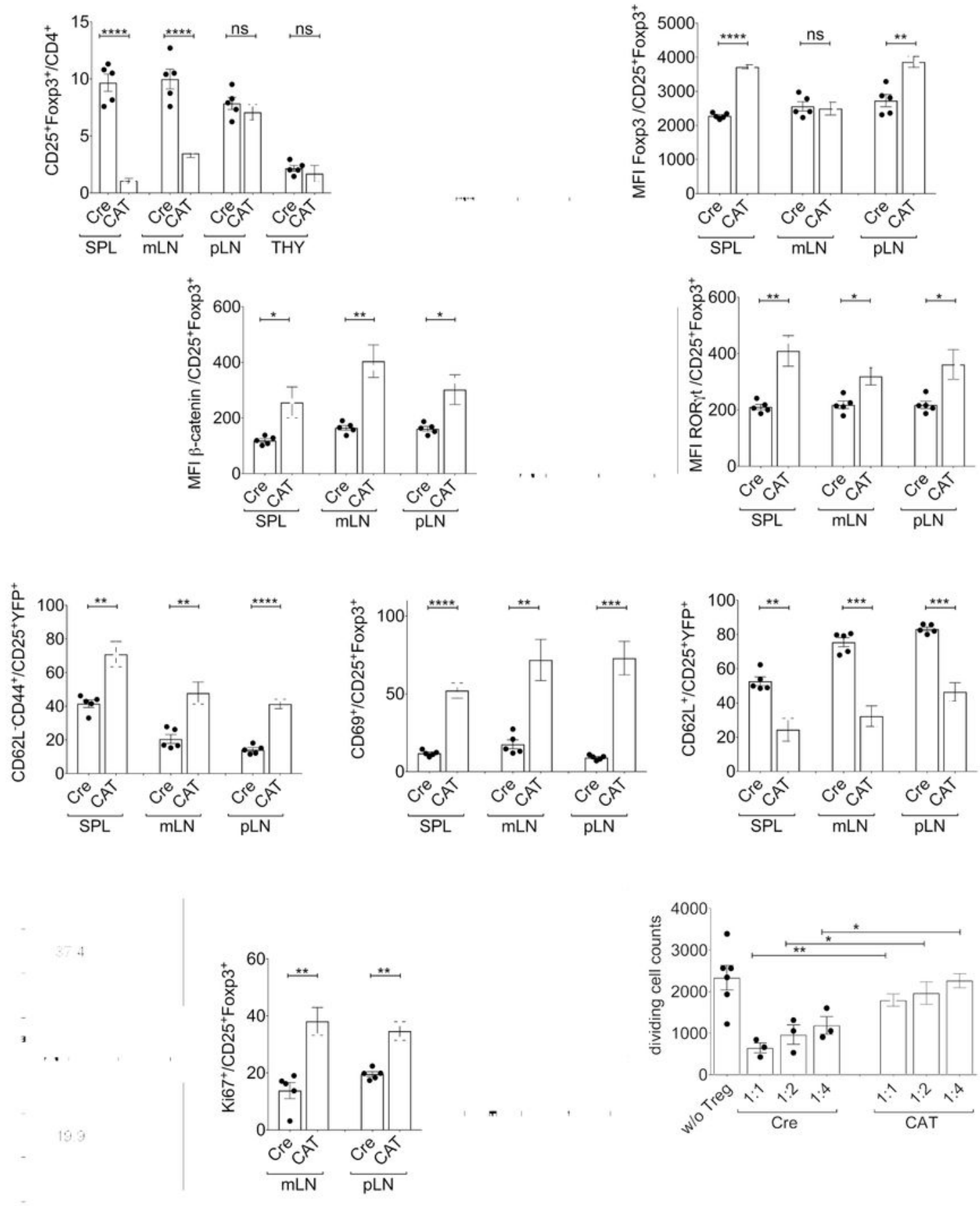

\section{Figure 4}

Treg-specific $\beta$-catenin stabilization results in a drastically altered, activated Treg phenotype in mice.a, Frequency of CD25+Foxp3+ Tregs in Cre and CAT mice within CD3+CD4+ T cells. Intracellular expression of, b, Foxp3, c, $\beta$-catenin and, d, RORyt in Foxp3Cre(0/+) wild-type (Cre) and Foxp3Cre(0/+) Ctnnb1fl(ex3) (CAT) Tregs cells are depicted as representative flow plots and cumulative column plots for peripheral lymphoid organs. e-g Activation status and proliferation of Treg cells in Cre and CAT mice was 
determined through staining and flow cytometric analysis of, e, CD44, f, CD69, g, CD62L and, h, Ki67 in the indicated lymphoid organs. g, Representative flow cytometric histograms and cumulative analysis of the suppressive capacity of Treg cells derived from Cre versus CAT mice. Fraction of proliferating, polyclonally activated CD4+ Tcon cells are shown. 1:1, 1:2, (...) = Treg:Tcon-ratio. pLN = peripheral lymph nodes, $\mathrm{mLN}=$ mesenteric lymph nodes, $\mathrm{SPL}=$ spleen; $\mathrm{n}(\mathrm{CAT})=3-10, \mathrm{n}(\mathrm{WT})=5-10$, statistical testing: unpaired t-test, error analysis: SEM
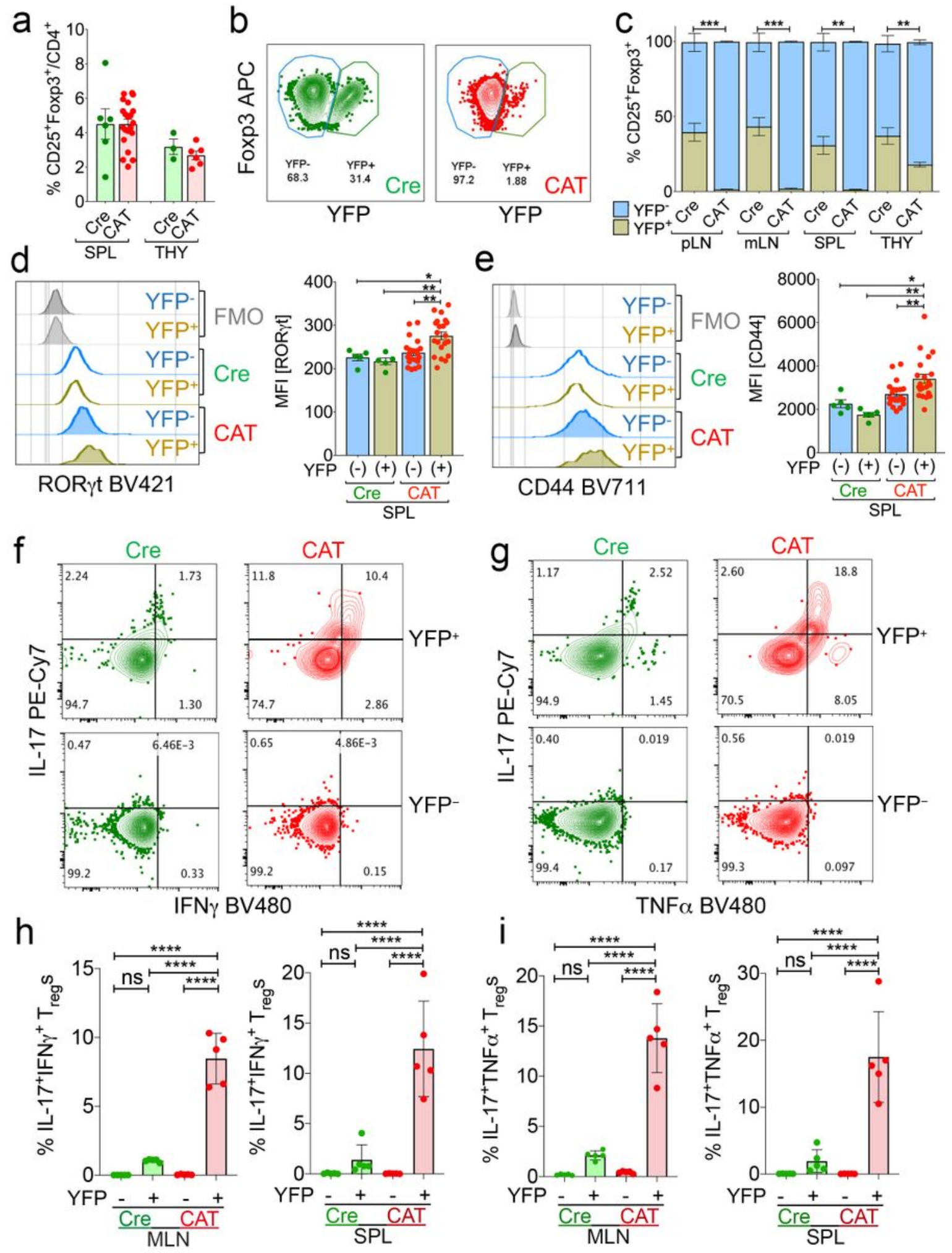

TNF $\alpha$ BV480
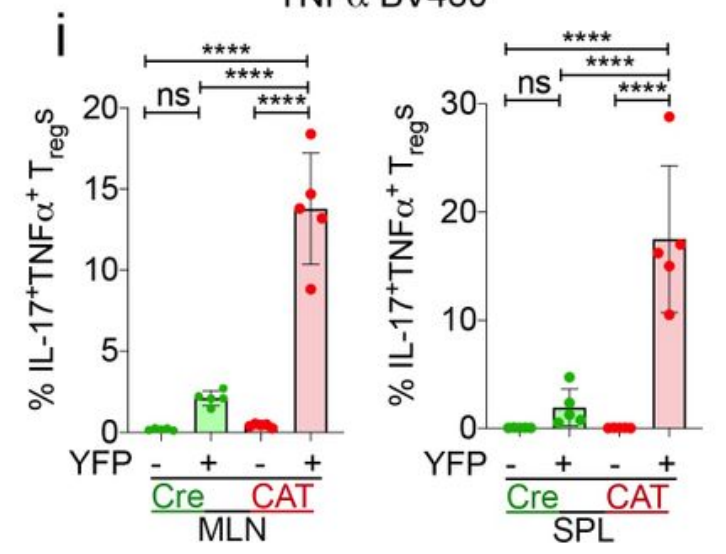

Figure 5 
$\beta$-cateninhigh Tregs have a competitive disadvantage in an unperturbed chimeric setting Flow cytometric characterization of Foxp3Cre(+/-) Ctnnb1fl(ex3) (CAT) and Foxp3Cre(+/-) (Cre) heterozygote, female mice representing intrinsic Treg chimeras. a, Frequency of Tregs within CD4+ T cells. b, Representative flowcytometric dot plots and, c, quantification of Cre+/YFP+ and Cre-/YFP- fractions in lymphoid organs. Representative flow cytometric histograms and cumulative analyses of $d$, RORyt and, e, CD44 expression in YFP+/YFP- Tregs. (FMO = fluorescence minus one negative staining control). Representative flowcytometric dot plots of $\mathrm{f}$, IL-17 versus IFNy and, g, IL-17 versus TNFa production in YFP+ versus YFP- Cre and CAT MLN Tregs. Cumulative analysis of the frequencies of coproduction of $h, I L-17$ and IFNy and, $i$, IL-17 and TNFa in YFP+ and YFP- Cre and CAT Tregs in MLNs and Spleen, as indicated. (statistical testing: unpaired t-test, error analysis: SEM). 
a

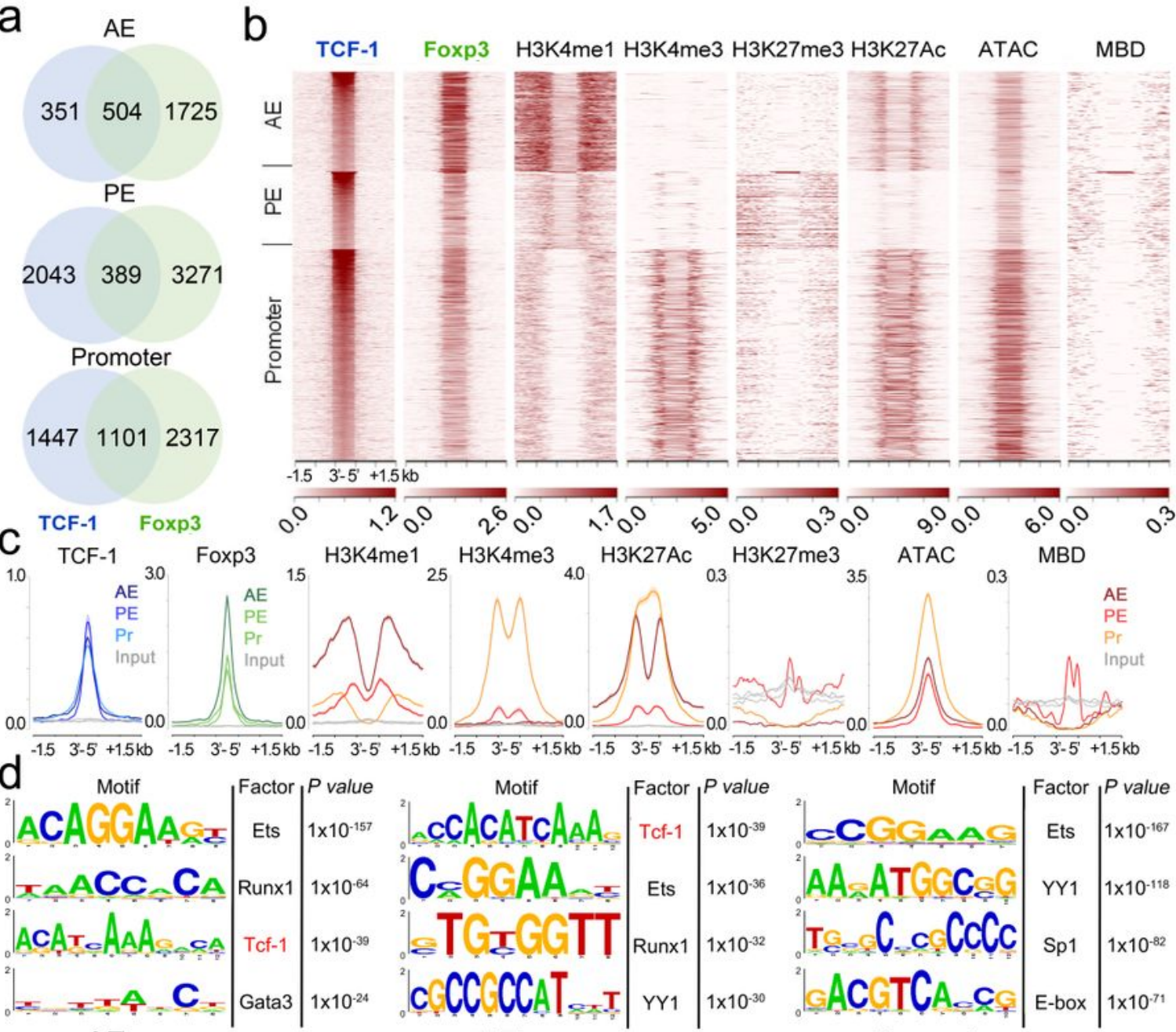

$\mathrm{AE}$

e

PE
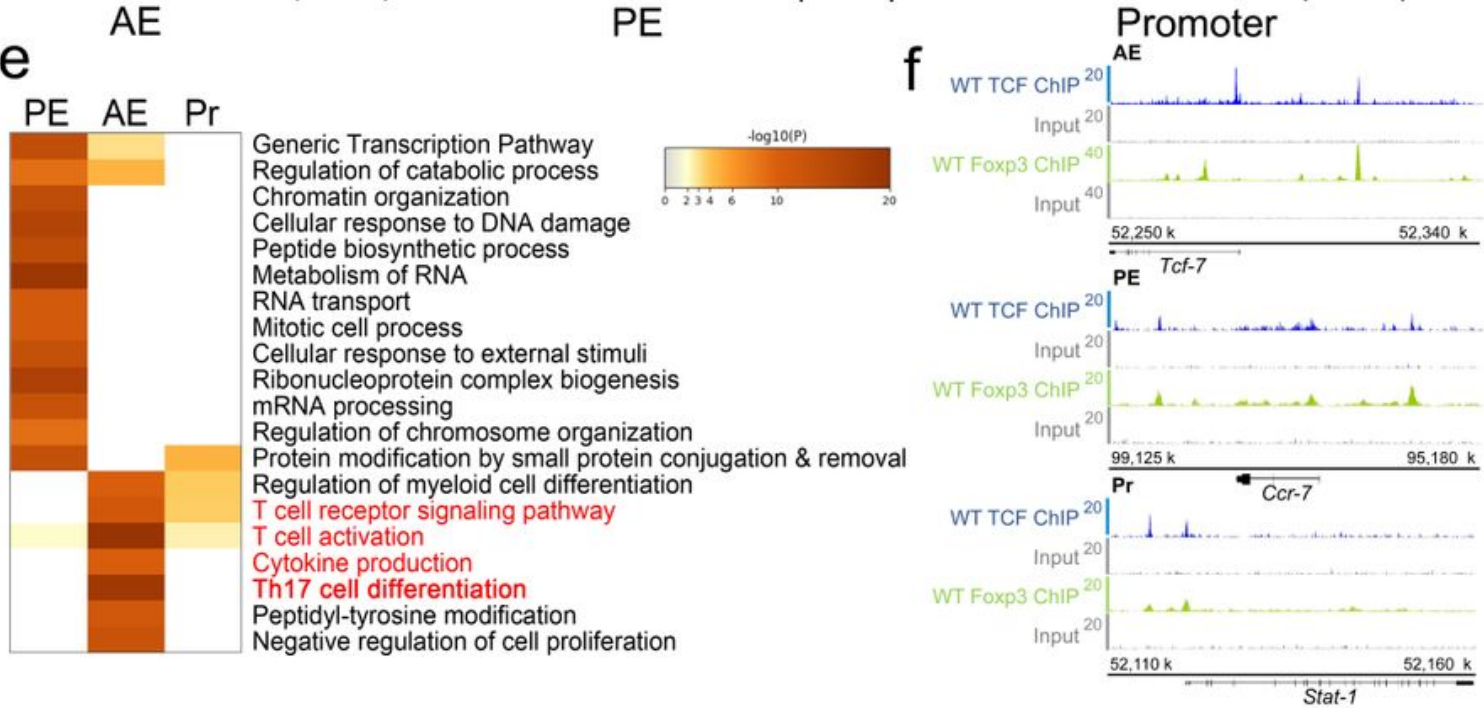

Figure 6

$\beta$-catenin DNA-binding partner TCF-1 co-binds accessible chromatin together with Foxp3 at gene loci crucial for bona fide Treg integrity and function. a, Venn diagrams of overlapping TCF-1 (blue) and Foxp3 (green) binding sites (numbers of peaks) in the genomic regions identified in Fig. S6 and S7 for TCF-1 and Foxp3, respectively, in Tregs ( $\mathrm{E} E=$ active enhancer, $\mathrm{PE}=$ poised enhancer, $\mathrm{Pr}=$ Promoter). $b$, Heat map of TCF-1/Foxp3 co-bound genomic regions ( $\pm 1.5 \mathrm{~kb}$ ). Enrichment of histone marks (H3K4me1, H3K4me3, 
H3K27me3, H3K27Ac), chromatin accessibility (ATAC) and DNA methylation (MBD) are shown. c, Comparative enrichment histograms of transcription factor binding (TCF-1, Foxp3), histone marks, chromatin accessibility, and DNA methylation marks at TCF-1/Foxp3 co-bound sites ( $\pm 1.5 \mathrm{~kb})$ in the indicated genomic regions established above. $d$, De novo transcription-factor-binding motif analysis (HOMER) of TCF-1/Foxp3 co-bound sites in the indicated genomic regions. Most highly significantly enriched motifs and corresponding $p$ values are listed. e, Functional pathways enriched for TCF-1/Foxp3 co-bound genes in the indicated genomic regions. Pathways and their statistical enrichment were determined using Metascape (http://www.metascape.org). f, Representative TCF-1/Foxp3 co-bound regions at the indicated loci (IGB=Integrated Genome Browser tracks) for the different genomic regions. ChIP-seq enrichment tracks for TCF-1, Foxp3, and respective Input controls are shown. 
a
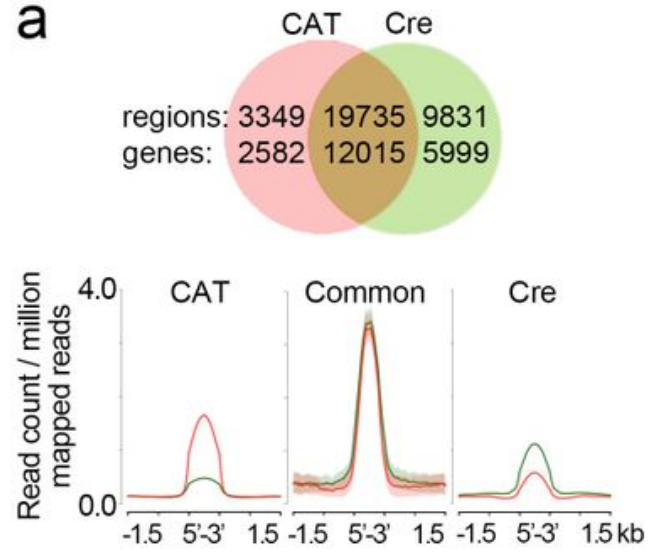

b

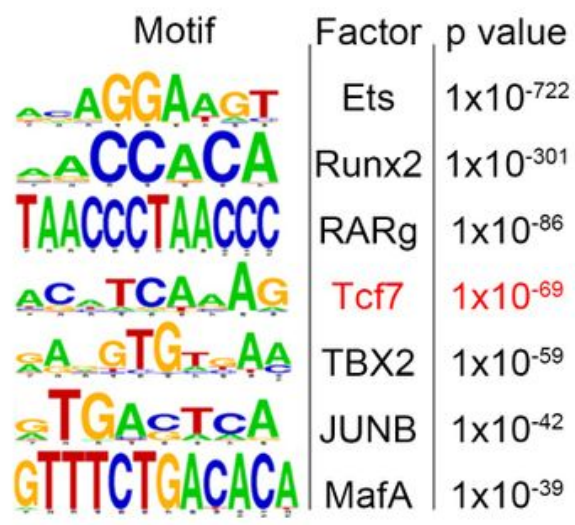

C

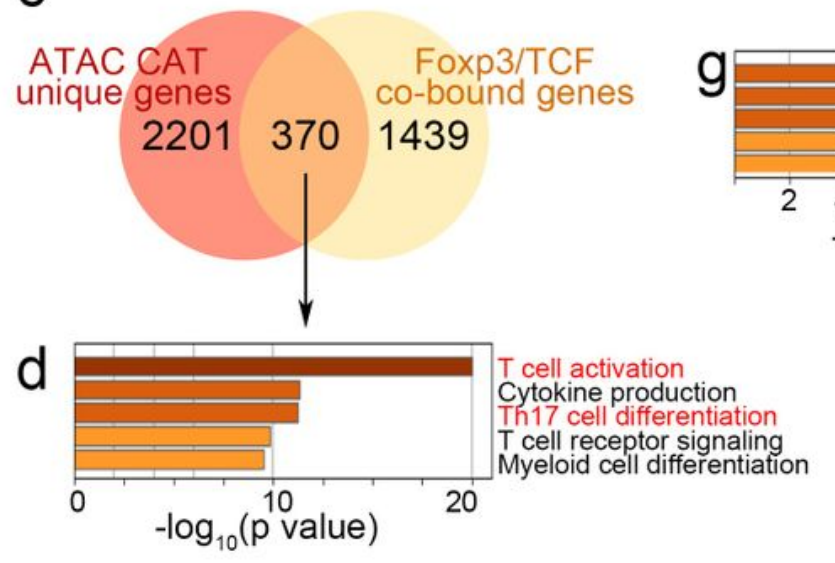

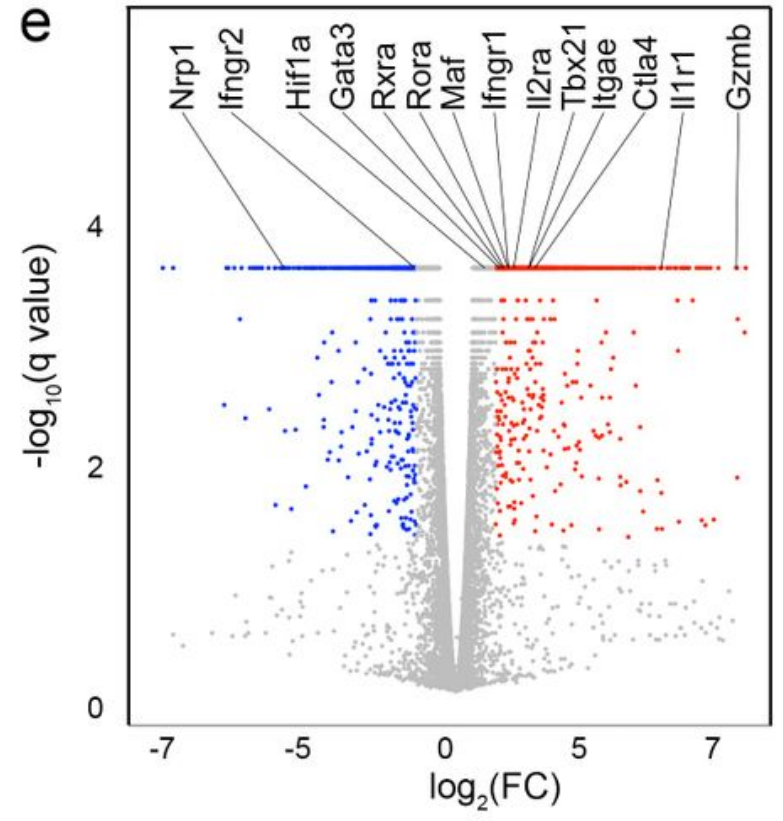
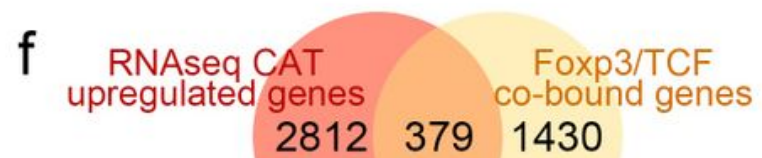

\section{Figure 7}

Activation of $\beta$-catenin in Tregs increased the accessibility and transcriptional activation of genes in Th17 differentiation and $T$ cell activation pathways. a, Venn diagrams comparing accessible chromatin sites in CAT versus Cre Tregs and comparative enrichment histograms of chromatin accessibility at CAT and Cre common sites, newly gained in CAT sites, as well as Cre unique sites. b, De novo transcription-factorbinding motif analysis (HOMER) of newly accessible sites in CAT Tregs. Most, significantly enriched 
motifs and corresponding $\mathrm{p}$ values are listed. c, Venn diagram comparing genes gaining newly accessible sites in CAT Tregs to TCF-1/Foxp3 co-bound genes. d, Functional pathway enrichment analysis (http://www.metascape.org) of genes that gain accessibility in CAT Tregs and are TCF-1/Foxp3 cobound. e, Volcano plot of differentially expressed genes in CAT Tregs over Cre Tregs (q-value cutoff < $1 \times 10-5)$. $f$, Venn diagram comparing genes that are significantly upregulated $(q<0.05)$ in CAT Tregs to TCF-1/Foxp3 co-bound genes. g, Functional pathway enrichment analysis (http://www.metascape.org) of genes that are transcriptionally up-regulated in CAT Tregs and co-bound by TCF-1/Foxp3. Cells for ATACseq and RNAseq analysis were isolated from pLNs of aged matched $21 \mathrm{~d}$ old CAT and Cre WT mice. 


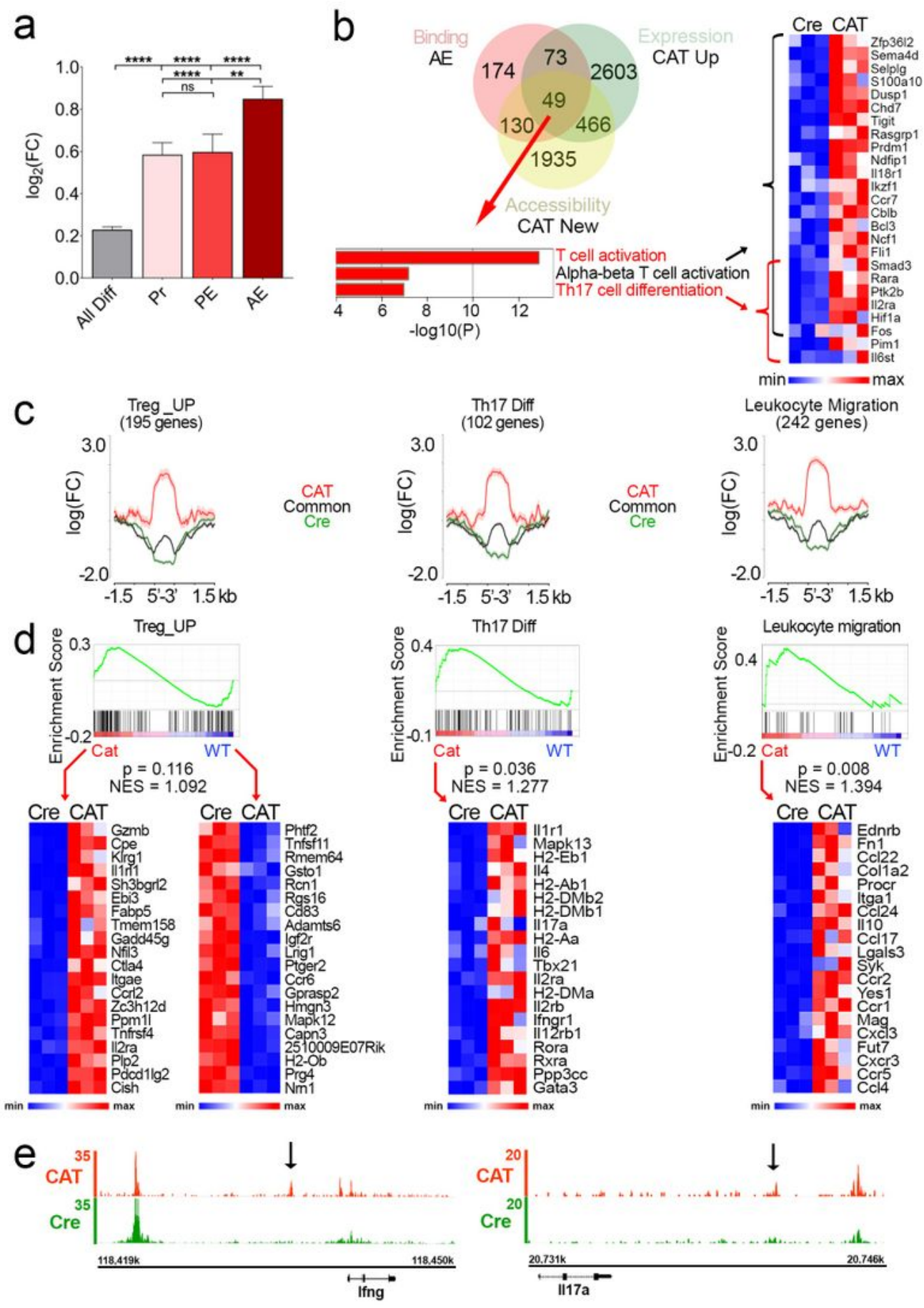

\section{Figure 8}

$\beta$-catenin stabilization epigenetically changes the activation of critical genes co-regulated by Foxp3 and TCF-1 to drive the observed phenotype of RORyt+ Tregs. a, Differential accessibility estimated by ATACseq in regulatory regions ( $\mathrm{AE}=$ active enhancer, $\mathrm{PE}=$ =poised enhancer, $\mathrm{Pr}=\mathrm{Promoter}$ ) of TCF-1/Foxp3 cobound genes compared to all differentially accessible genes (All Diff) (Mann-Whitney test). b, Venn diagram comparing TCF-1/Foxp3 co-bound genes in active enhancers (red), transcriptionally up-regulated 
genes in CAT Tregs (green), and genes that gained newly accessible chromatin in CAT Tregs (yellow) . Functional pathway enrichment analysis (http://www.metascape.org) of the Venn diagram overlap and corresponding RNA expression heat map ( $\mathrm{n}=3$ biological replicates for each genotype, FPKMs). c, Differential accessibility at genes within the Treg_UP, Th17 differentiation, and leukocyte migration signatures. d, Gene-set enrichment analysis (GSEA) (top) and associated RNA expression heatmaps of indicated leading-edge genes (bottom). ( $n=3$ biological replicates for each genotype, FPKMs) e, Integrated Genome Browser tracks for ATACseq accessibility at IFN- $\gamma$ and IL17a genomic loci in CAT and Cre Tregs. Arrows indicate CAT-enriched open chromatin peaks.

\section{Supplementary Files}

This is a list of supplementary files associated with this preprint. Click to download.

- QuandtetalSupplementaryTable1.xlsx

- QuandtetalSupplementaryTable2.xIsx 